\title{
THE RELATION BETWEEN GALAXY ACTIVITY AND THE DYNAMICS OF COMPACT GROUPS OF GALAXIES
}

\author{
R. Coziol \\ Departamento de Astronomía, Universidad de Guanajuato, Apdo. Postal 144, 36000 Guanajuato, Mexico; rcoziol@astro.ugto.mx \\ E. BRINKS \\ Instituto Nacional de Astrofísica, Optica y Electrónica, Apdo. Postal 51 y 216, 72000, Puebla Mexico; ebrinks@inaoep.mx \\ AND \\ H. Bravo-Alfaro \\ Departamento de Astronomía, Universidad de Guanajuato, Apdo. Postal 144, 36000 Guanajuato, Mexico; hector@astro.ugto.mx \\ Received 2004 January 23; accepted 2004 April 2
}

\begin{abstract}
Using a sample of 91 galaxies distributed over 27 compact groups (CGs) of galaxies, we define an index that allows us to quantify their level of activity due to an active galactic nucleus (AGN) or star formation. By combining the mean activity index with the mean morphological type of the galaxies in a group, we are able to quantify the evolutionary state of the groups. We find that they span an evolutionary sequence that correlates with the spatial configuration of the galaxies in the CG. We distinguish three main configuration types: A, B, and C. Type A CGs show predominantly low velocity dispersions and are rich in late-type spirals that show active star formation or harbor an AGN. Type B groups have intermediate velocity dispersions and contain a large fraction of interacting or merging galaxies. Type $\mathrm{C}$ comprises CGs with high velocity dispersions, which are dominated by elliptical galaxies that show no activity. We suggest that evolution proceeds $\mathrm{A} \Rightarrow \mathrm{B} \Rightarrow \mathrm{C}$. Mapping the groups with different evolution levels in a diagram of radius versus velocity dispersion does not reveal the pattern expected based on the conventional fast merger model for CGs, which predicts a direct relation between these two parameters. Instead, we observe a trend contrary to expectation: the evolutionary state of a group increases with velocity dispersion. This trend seems to be related to the masses of the structures in which CGs are embedded. In general, the evolutionary state of a group increases with the mass of the structure. This suggests either that galaxies evolve more rapidly in massive structures or that the formation of CGs embedded in massive structures predated the formation of CGs associated with lower mass systems. Our observations are consistent with the structure formation predicted by the CDM model (or $\Lambda \mathrm{CDM}$ ), only if the formation of galaxies is a biased process.
\end{abstract}

Key words: galaxies: evolution — galaxies: formation — galaxies: interactions

\section{INTRODUCTION}

Although it seems today an inescapable conclusion that the formation and evolution of galaxies is influenced by their environment, the details of how these processes occur in space and time are still largely unknown. One example is that of compact groups (CGs) of galaxies. As a result of our studies of the activity in galaxies in CGs, we now have a better understanding of the evolution of galaxies in these systems (Coziol et al. 1998a, 1998b, 2000). Our observations showed emission-line galaxies to be remarkably frequent, representing more than $50 \%$ of the galaxies in CGs. Nonthermal activity, in the form of Seyfert 2 galaxies, LINERs, and numerous lowluminosity active galactic nuclei (LLAGNs; see Coziol et al. 1998b for a definition of this activity type in CGs), was found to constitute one important aspect of this activity, whereas nuclear star formation, although mildly enhanced in some groups, was noted to be generally declining. These observations were considered to be consistent with the effects of tidal forces exerted on disk galaxies when they fall into the potential well of a rich cluster or group of galaxies (Coziol et al. 2000). Tidal stripping removes gas from a galaxy, reducing star formation in the disk, whereas tidal triggering starts a short burst of star formation in the nuclear region and fuels an AGN (Merritt 1983, 1984; Byrd \& Valtonen 1990; Henriksen \& Byrd 1996; Fujita 1998).
The above processes may also produce the densitymorphology-activity relation observed in CGs (Coziol et al. 1998b). As they lose their gas and form new stars near their nucleus, thus increasing their bulge, the morphology of spiral galaxies falling into groups is transformed to an earlier type. Assuming that groups are continuously replenished by spiral galaxies from the field (Governato et al. 1996; Coziol et al. 2000), the cores of the groups are naturally expected to be populated by quiescent galaxies and AGNs (LLAGNs included), all having an early-type morphology, and their periphery to be richer in late-type star-forming galaxies.

What is missing in the above description is a connection between galaxy evolution and the physical processes responsible for the formation and evolution of CGs. Our first interpretation of these systems, based on galaxy-galaxy interaction models, suggested that they could not survive mergers over a long period of time (Barnes 1989), which seems in conflict with the high number of CGs observed today (for a different point of view, see Aceves \& Velázquez 2002). It was then realized that this paradox could be explained, in part, by the fairly simplistic assumptions made about the nature of these systems. For example, if CGs are associated with larger and more dynamically complex structures, as many redshift surveys suggest (Rood \& Struble 1994; Ramella et al. 1994; Garcia 1995; Ribeiro et al. 1998; Barton et al. 1998), their formation and evolution must also be more complicated than previously thought. 
In this study, we examine further the question of the formation and evolution of CGs, by extending our analysis of the activity to a larger sample of Hickson compact groups of galaxies (HCGs; Hickson 1982).

\section{OBSERVATIONS}

Long-slit spectra of 65 galaxies in 19 HCGs were obtained with the $2.1 \mathrm{~m}$ telescope located at San Pedro Mártir in Baja California during one run of four nights in 2002 April and another of five nights in 2002 October. A Boller \& Chivens spectrograph was used in conjunction with a SITE $1024 \times 1024$ CCD, yielding a plate scale of 1".05 per pixel. During the observations, the slit width was kept open to $240 \mu \mathrm{m}$, which corresponds to 3"1 1 on the sky. This aperture was chosen to be slightly larger than the effective seeing of $2^{\prime \prime}$ (which includes dome seeing and instrument response). We used a 300 line $\mathrm{mm}^{-1}$ grating, blazed at $5000 \AA$, which, with the slit width adopted, yielded an average resolution of $8 \AA$ Av over a $4096 \AA$ range.

Table 1 shows an excerpt of our observation logbook. Column (1) gives the date of observation; column (2) identifies the object (HCG numbers and letters, as given by Hickson 1982 and as archived in catalog VII/213 at the Centre de Données astronomiques de Strasbourg [CDS]). The positions of the galaxies observed in columns (3) and (4) are those given in the CDS catalog, precessed by us to the year 2000. The heliocentric velocities, column (5), and the morphologies of the observed galaxies, columns (6) and (7), were also taken from the same source. Only galaxies pertaining to the groups (based on their redshifts) were observed. Note that, because of observational constraints, we did not succeed in observing all the galaxies in each group, as originally planned. Our sample is therefore somewhat incomplete and biased toward the most luminous galaxies in each group. The effect this bias might have on our analysis is discussed at length in $\S 4.3$.

Column (8) of Table 1 gives the number of exposures and their durations. In general, we took three spectra of $900 \mathrm{~s}$ each. The long slit (covering $322^{\prime \prime}$ on the sky) was always centered on the most luminous part of the galaxies and usually aligned in the east-west direction to minimize errors introduced by guiding. In a very few cases, the slit was rotated to a different angle to put more than one galaxy in the slit. These cases are identified by a plus sign in front of the HCG number of the galaxy. The effective air mass is given in the last column. Most of the observations were performed under low-air-mass conditions, minimizing differential diffraction.

Before the first exposure, and after each of the subsequent ones, a He-Ar lamp was observed for wavelength calibration. During each night, between two and three standard stars were observed to flux calibrate the spectra. During our observations we noted the frequent passage of light clouds during the night or the presence of cirrus at the beginning or end of the night, which suggests that the conditions were not photometric. Absolute flux calibration, however, is not critical for our analysis.

\section{RESULTS}

\subsection{Reduction and Template Subtraction}

Standard reduction techniques were followed using IRAF. ${ }^{1}$ After bias subtraction, the spectra were divided by a normal-

\footnotetext{
${ }^{1}$ IRAF, the Image Reduction and Analysis Facility, a general purpose software system for the reduction and analysis of astronomical data, is written and supported by the IRAF programming group at the National Optical Astronomy Observatories in Tucson, Arizona (http://iraf.noao.edu).
}

ized dome flat. The sky was subtracted before reducing the spectra to one dimension. After calibrating the one-dimensional spectra in wavelength, the spectra were flux calibrated. The uncertainty in the flux calibration is estimated to be $10 \%$. For each galaxy, an average spectrum was obtained by combining the complete set of one-dimensional spectra (usually three) available for that object.

The apertures used for the reduction to one-dimensional spectra contain $99 \%$ of the light from the most luminous part of the galaxies (the nucleus). The equivalent linear size for the spatial apertures used are listed in column (2) of Table 2. It is interesting to note how compact the light distribution is in all these galaxies. For the emission-line galaxies in particular, such compactness suggests that the ionized gas covers regions extending out to only a few kpc around the nucleus (the only exception being HCG 56a). A comparison with the $\mathrm{H} \alpha$ imaging study of Severgnini et al. (1999), which lists the isophotal fluxes and luminosities of $\mathrm{H} \alpha+\left[\mathrm{N}_{\mathrm{II}}\right]$ in an area $1 \sigma$ above the background for a sample of 73 HCGs, suggests that our spectroscopic survey may have missed at most a few regions of low star formation activity in the disk of some galaxies. Such activity does not seem to be predominant in CGs.

Some $75 \%$ of the emission-line galaxies in the groups show strong Balmer absorption lines, which need to be corrected for before measuring line ratios in these galaxies. To accomplish this we subtracted four different templates, using the following quiescent galaxies from our sample: HCG 10b, 15c, 30a, and $58 \mathrm{~d}$. Since we are interested only in classifying the galaxies, we did not try to fit the templates to the entire candidate galaxy spectrum. Instead, we concentrated on obtaining a good fit for the Balmer lines. The method followed is straightforward and easy to apply. First, all spectra are corrected to zero redshift. Then a region $400 \AA$ wide around $\mathrm{H} \alpha$ and $1500 \AA$ wide around $\mathrm{H} \beta$ is selected to fit the continuum on each side of the lines. Note that a larger wavelength range is needed for $\mathrm{H} \beta$ in order to bridge the strong $\mathrm{Mg}$ absorption bands visible in this region of the spectrum. Each spectrum is then normalized, dividing it by the fitted continuum. The templates, which were normalized the same way, are then simply subtracted from the candidate galaxies. One example of a template subtraction is shown in Figure 1.

The emission lines are measured in the template-subtracted spectrum by integrating the flux under the lines. For $\mathrm{H} \alpha$, the IRAF deblend routine in SPLOT was used to correct for any contribution from [ $\mathrm{N}$ II]. We only determined line fluxes in those cases in which after template subtraction the line flux is larger than the rms in the residual (at full resolution). The $\mathrm{H} \alpha$ flux is usually higher than $3 \sigma$, and is higher than or equal to $2 \sigma$ for $\mathrm{H} \beta$. In 10 cases only 1 of the lines (mostly $\mathrm{H} \alpha$ ) was detected after template subtraction. For the line ratios, we calculated the mean of four values measured after subtracting one by one each of the individual four templates. We adopted the dispersion of the mean as the uncertainty. The two most important line ratios $[\mathrm{N}$ II $] / \mathrm{H} \alpha$ and $[\mathrm{O}$ III $] / \mathrm{H} \beta$ are given in Table 2. A plus sign in front of the HCG number identifies the few emission-line galaxies for which a template subtraction was not deemed necessary, as visual inspection of the spectra did not show any signs of underlying absorption. Note that we did not apply any correction for dust extinction, since these two line ratios are known to be insensitive to this (Veilleux \& Osterbrock 1987). In Table 2, we also give the $\mathrm{H} \alpha$ luminosity and equivalent width (EW) of the $\mathrm{H} \alpha+[\mathrm{N}$ II] lines as measured in the template-subtracted spectra. The uncertainty for 
TABLE 1

Log of the Observations and Main Characteristics of the Targets

\begin{tabular}{|c|c|c|c|c|c|c|c|c|}
\hline $\begin{array}{l}\text { Observing Date } \\
\text { (1) }\end{array}$ & $\begin{array}{l}\text { HCG } \\
(2)\end{array}$ & $\begin{array}{c}\alpha \\
(2000) \\
(3)\end{array}$ & $\begin{array}{c}\delta \\
(2000) \\
(4)\end{array}$ & $\begin{array}{c}v_{r} \\
\left(\mathrm{~km} \mathrm{~s}^{-1}\right) \\
(5)\end{array}$ & $\begin{array}{c}T \\
(6)\end{array}$ & $\begin{array}{l}\text { Morphology } \\
\text { (7) }\end{array}$ & $\begin{array}{c}\text { Exposure Time } \\
(\times 900 \mathrm{~s}) \\
(8)\end{array}$ & $\begin{array}{c}\text { Air Mass } \\
\text { (9) }\end{array}$ \\
\hline 2002 Oct $16 \ldots \ldots \ldots$ & $8 \mathrm{a}$ & 004934.26 & 233440.14 & 16014 & -5 & E5 & 3 & 1.0 \\
\hline 2002 Oct $16 \ldots \ldots \ldots$ & $+8 \mathrm{~b}$ & 004935.29 & 233528.32 & 15966 & -2 & S0 & 3 & 1.2 \\
\hline 2002 Oct $16 \ldots \ldots \ldots$ & $+8 \mathrm{c}$ & 004935.90 & 233502.01 & 17087 & -2 & S0 & 3 & 1.2 \\
\hline 2002 Oct $16 \ldots \ldots \ldots$ & $8 \mathrm{~d}$ & 004936.77 & 233422.80 & 16341 & -2 & S0 & 3 & 1.0 \\
\hline 2002 Oct $17 \ldots \ldots \ldots$ & $10 \mathrm{a}$ & 012621.43 & 344207.51 & 5148 & 3 & $\mathrm{SBb}$ & 3 & 1.0 \\
\hline 2002 Oct $17 \ldots \ldots \ldots$ & $10 \mathrm{~b}$ & 012540.33 & 344246.39 & 4862 & -5 & E1 & 3 & 1.1 \\
\hline 2002 Oct $20 \ldots \ldots \ldots$ & $10 \mathrm{c}$ & 012618.81 & 344514.38 & 4660 & 5 & Sc & 3 & 1.0 \\
\hline 2002 Oct $20 \ldots \ldots \ldots$ & $10 \mathrm{~d}$ & 012630.84 & 344030.46 & 4620 & 6 & Scd & 3 & 1.1 \\
\hline 2002 Oct $18 \ldots \ldots \ldots$ & $15 \mathrm{a}$ & 020753.00 & 021003.31 & 6967 & 1 & $\mathrm{Sa}$ & 3 & 1.2 \\
\hline 2002 Oct $19 \ldots \ldots \ldots$ & $15 \mathrm{~b}$ & 020734.09 & 020654.73 & 7117 & -5 & E0 & 3 & 1.1 \\
\hline 2002 Oct $19 \ldots \ldots \ldots$ & $15 \mathrm{c}$ & 020739.75 & 020859.02 & 7222 & -5 & E0 & 3 & 1.1 \\
\hline 2002 Oct $19 \ldots \ldots \ldots$ & $15 \mathrm{~d}$ & 020737.49 & 021050.80 & 6244 & -5 & E2 & 3 & 1.2 \\
\hline 2002 Oct $19 \ldots \ldots \ldots$ & $30 \mathrm{a}$ & 043618.57 & -024952.67 & 4697 & 5 & $\mathrm{SBc}$ & 3 & 1.2 \\
\hline 2002 Oct $19 \ldots \ldots \ldots$ & $30 \mathrm{~b}$ & 043630.27 & -025159.66 & 4625 & 1 & $\mathrm{Sa}$ & 3 & 1.2 \\
\hline 2002 Oct $20 \ldots \ldots \ldots$ & $31 \mathrm{a}$ & 050138.74 & -041534.11 & 4042 & 8 & $\mathrm{Sdm}$ & 4 & 1.2 \\
\hline 2002 Oct $17 \ldots \ldots \ldots$ & $34 \mathrm{a}$ & 052145.93 & 064119.73 & 8997 & -5 & E2 & 3 & 1.1 \\
\hline 2002 Oct $17 \ldots \ldots \ldots$ & $34 \mathrm{c}$ & 052148.96 & 064054.71 & 9392 & 6 & SBd & 1 & 1.2 \\
\hline 2002 Apr 8............. & $37 \mathrm{a}$ & 091339.44 & 295932.61 & 6745 & -3 & E7 & 3 & 1.0 \\
\hline 2002 Apr $8 \ldots \ldots \ldots \ldots$ & $+37 \mathrm{~b}$ & 091332.60 & 295959.04 & 6741 & 4 & $\mathrm{Sbc}$ & 6 & 1.0 \\
\hline 2002 Apr $8 \ldots \ldots \ldots \ldots$ & $+37 \mathrm{c}$ & 091337.22 & 295958.22 & 7357 & 0 & S0a & 6 & 1.0 \\
\hline 2002 Apr $8 \ldots \ldots \ldots \ldots$ & $37 d$ & 091333.84 & 300051.78 & 6207 & 8 & SBdm & 3 & 1.1 \\
\hline 2002 Apr $10 \ldots \ldots \ldots$ & $40 \mathrm{a}$ & 093853.52 & -045056.53 & 6628 & -5 & E3 & 3 & 1.2 \\
\hline 2002 Apr $10 \ldots \ldots \ldots$ & $40 \mathrm{~b}$ & 093855.04 & -045157.90 & 6842 & -2 & S0 & 3 & 1.2 \\
\hline 2002 Apr $10 \ldots \ldots \ldots$ & $40 \mathrm{c}$ & 093853.20 & -045133.72 & 6890 & 4 & $\mathrm{Sbc}$ & 3 & 1.3 \\
\hline 2002 Apr $10 \ldots \ldots \ldots$ & $40 \mathrm{~d}$ & 093855.77 & -045014.53 & 6492 & 1 & $\mathrm{SBa}$ & 3 & 1.3 \\
\hline 2002 Apr $10 \ldots \ldots \ldots$ & $40 \mathrm{e}$ & 093855.45 & -045127.91 & 6625 & 5 & $\mathrm{Sc}$ & 3 & 1.2 \\
\hline 2002 Apr $9 \ldots \ldots \ldots . .$. & $+54 \mathrm{a}$ & 112915.17 & 203500.73 & 1397 & 8 & $\mathrm{Sdm}$ & 4 & 1.0 \\
\hline 2002 Apr $9 \ldots \ldots \ldots . .$. & $+54 \mathrm{~b}$ & 112914.05 & 203453.24 & 1412 & 10 & $\operatorname{Im}$ & 4 & 1.0 \\
\hline 2002 Apr $9 \ldots \ldots \ldots . .$. & $54 \mathrm{c}$ & 112916.26 & 203511.12 & 1420 & 10 & $\operatorname{Im}$ & 3 & 1.0 \\
\hline 2002 Apr $9 \ldots \ldots \ldots . .$. & $54 \mathrm{~d}$ & 112916.50 & 203518.82 & 1670 & 10 & $\operatorname{Im}$ & 3 & 1.1 \\
\hline 2002 Apr $8 \ldots \ldots \ldots \ldots$ & $+56 \mathrm{a}$ & 113246.64 & 525627.03 & 8245 & 5 & Sc & 3 & 1.5 \\
\hline 2002 Apr $8 \ldots \ldots \ldots . .$. & $+56 \mathrm{~b}$ & 113240.45 & 525701.59 & 7919 & -2 & SB0 & 5 & 1.1 \\
\hline 2002 Apr $8 \ldots \ldots \ldots . .$. & $56 \mathrm{c}$ & 113236.69 & 525651.03 & 8110 & -2 & S0 & 3 & 1.1 \\
\hline 2002 Apr 8............ & $56 \mathrm{~d}$ & 113235.29 & 525649.84 & 8346 & -2 & S0 & 3 & 1.2 \\
\hline 2002 Apr $8 \ldots \ldots \ldots . .$. & $56 \mathrm{e}$ & 113232.73 & 525620.96 & 7924 & -2 & S0 & 3 & 1.3 \\
\hline 2002 Apr $10 \ldots \ldots \ldots$ & $58 \mathrm{a}$ & 114211.06 & 101639.62 & 6138 & 3 & $\mathrm{Sb}$ & 3 & 1.1 \\
\hline 2002 Apr $10 \ldots \ldots \ldots$ & $58 \mathrm{~b}$ & 114223.56 & 101551.05 & 6503 & 2 & SBab & 3 & 1.1 \\
\hline 2002 Apr $10 \ldots \ldots \ldots$ & $58 \mathrm{c}$ & 114153.15 & 101814.63 & 6103 & 0 & SB0a & 3 & 1.2 \\
\hline 2002 Apr $10 \ldots \ldots \ldots$ & $58 \mathrm{~d}$ & 114205.89 & 102103.06 & 6270 & -5 & E1 & 3 & 1.2 \\
\hline 2002 Apr $10 \ldots \ldots \ldots$ & $58 \mathrm{e}$ & 114204.82 & 102301.86 & 6052 & 4 & $\mathrm{Sbc}$ & 3 & 1.4 \\
\hline 2002 Apr $9 \ldots \ldots \ldots . .$. & $68 \mathrm{a}$ & 135326.53 & 401658.35 & 2162 & -2 & S0 & 3 & 1.0 \\
\hline 2002 Apr $9 \ldots \ldots \ldots . .$. & $68 b$ & 135326.62 & 401808.65 & 2635 & -5 & $\mathrm{E} 2$ & 3 & 1.0 \\
\hline 2002 Apr $9 \ldots \ldots \ldots . .$. & $68 \mathrm{c}$ & 135321.69 & 402147.68 & 2313 & 4 & $\mathrm{SBbc}$ & 4 & 1.1 \\
\hline 2002 Apr $8 \ldots \ldots \ldots . .$. & $74 \mathrm{a}$ & 151924.91 & 205344.17 & 12255 & -5 & E1 & 3 & 1.1 \\
\hline 2002 Apr $9 \ldots \ldots \ldots . .$. & $74 \mathrm{~b}$ & 151924.38 & 205323.44 & 12110 & -5 & E3 & 3 & 1.1 \\
\hline 2002 Apr $9 \ldots \ldots \ldots . .$. & $74 \mathrm{c}$ & 151926.18 & 205356.44 & 12266 & -2 & S0 & 3 & 1.1 \\
\hline 2002 Apr $9 \ldots \ldots \ldots . .$. & $74 d$ & 151932.02 & 205257.47 & 11681 & -5 & E2 & 3 & 1.2 \\
\hline 2002 Apr 7 ............. & $79 a$ & 155911.41 & 204514.86 & 4292 & -5 & E0 & 3 & 1.0 \\
\hline 2002 Apr $7 \ldots \ldots \ldots \ldots$ & $79 b$ & 155912.61 & 204547.14 & 4446 & -2 & S0 & 3 & 1.0 \\
\hline 2002 Apr $7 \ldots \ldots \ldots . .$. & $79 \mathrm{c}$ & 155910.95 & 204541.43 & 4146 & -2 & S0 & 2 & 1.0 \\
\hline 2002 Apr $10 \ldots \ldots \ldots$ & $79 \mathrm{~d}$ & 155912.01 & 204447.20 & 4503 & 8 & $\mathrm{Sdm}$ & 3 & 1.1 \\
\hline 2002 Oct $17 \ldots \ldots \ldots$ & $88 b$ & 205229.69 & -054447.61 & 6010 & 3 & $\mathrm{SBb}$ & 4 & 1.2 \\
\hline 2002 Oct $17 \ldots \ldots \ldots$ & $88 \mathrm{c}$ & 205225.94 & -054620.12 & 6083 & 5 & $\mathrm{Sc}$ & 2 & 1.3 \\
\hline 2002 Oct $16 \ldots \ldots \ldots$ & $89 a$ & 212001.03 & -035520.26 & 8850 & 5 & Sc & 3 & 1.2 \\
\hline 2002 Oct $16 \ldots \ldots \ldots$ & $89 b$ & 212019.21 & -035346.81 & 8985 & 5 & $\mathrm{SBc}$ & 3 & 1.5 \\
\hline 2002 Oct $17 \ldots \ldots \ldots$ & $93 \mathrm{a}$ & 231515.98 & 185741.36 & 5140 & -5 & E1 & 3 & 1.0 \\
\hline 2002 Oct $17 \ldots \ldots \ldots$ & $93 b$ & 231517.17 & 190229.77 & 4672 & 6 & $\mathrm{SBd}$ & 3 & 1.0 \\
\hline 2002 Oct $17 \ldots \ldots \ldots$ & $93 \mathrm{c}$ & 231503.59 & 185823.28 & 5132 & 1 & $\mathrm{SBa}$ & 3 & 1.1 \\
\hline 2002 Oct $20 \ldots \ldots \ldots$ & $93 d$ & 231533.10 & 190252.50 & 5173 & -2 & SB0 & 3 & 1.0 \\
\hline 2002 Oct $18 \ldots \ldots \ldots$ & $+94 \mathrm{a}$ & 231713.44 & 184228.22 & 12040 & -5 & E1 & 3 & 1.0 \\
\hline 2002 Oct $18 \ldots \ldots \ldots$ & $+94 b$ & 231711.94 & 184203.30 & 11974 & -5 & E3 & 3 & 1.0 \\
\hline 2002 Oct $18 \ldots \ldots \ldots$ & $+94 \mathrm{c}$ & 231720.26 & 184403.62 & 12120 & -2 & S0 & 3 & 1.0 \\
\hline 2002 Oct $20 \ldots \ldots \ldots$ & $94 d$ & 231715.19 & 184242.55 & 13009 & -2 & S0 & 3 & 1.1 \\
\hline 2002 Oct $18 \ldots \ldots \ldots$ & $+98 \mathrm{a}$ & 235410.08 & 002258.07 & 7855 & -2 & SB0 & 5 & 1.2 \\
\hline 2002 Oct $18 \ldots \ldots \ldots$ & $+98 \mathrm{~b}$ & 235412.21 & 002235.88 & 7959 & -2 & S0 & 5 & 1.2 \\
\hline
\end{tabular}

Nоте.-Units of right ascension are hours, minutes, and seconds, and units of declination are degrees, arcminutes, and arcseconds. 
TABLE 2

Observed Line Ratios, Fluxes, and Equivalent Widths

\begin{tabular}{|c|c|c|c|c|c|c|c|}
\hline $\begin{array}{l}\mathrm{HCG} \\
(1)\end{array}$ & $\begin{array}{l}\text { Aperture } \\
(\mathrm{kpc}) \\
(2)\end{array}$ & 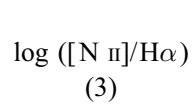 & $\begin{array}{c}\log \left(\left[\mathrm{O}_{\mathrm{III}}\right] / \mathrm{H} \beta\right) \\
(4)\end{array}$ & $\begin{array}{c}\log \left(L_{\mathrm{H} \alpha}\right) \\
\left(\operatorname{ergs~s}^{-1}\right) \\
(5)\end{array}$ & $\begin{array}{c}\mathrm{EW}(\mathrm{H} \alpha+\mathrm{N} \text { II }) \\
(\AA) \\
(6)\end{array}$ & $\begin{array}{c}\text { Activity Type } \\
\text { (7) }\end{array}$ & $\begin{array}{c}\mathrm{EW}_{\text {act }} \\
(\AA) \\
(8)\end{array}$ \\
\hline $8 \mathrm{a} \ldots \ldots \ldots \ldots \ldots$ & 12 & $\ldots$ & $\ldots$ & $\ldots$ & $\ldots$ & No emission & $\ldots$ \\
\hline 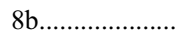 & 11 & $\ldots$ & $\ldots$ & $\ldots$ & $\ldots$ & No emission & $\ldots$ \\
\hline $8 \mathrm{c} \ldots \ldots \ldots \ldots \ldots \ldots$ & 10 & $\ldots$ & $\ldots$ & $\ldots$ & $\ldots$ & No emission & $\ldots$ \\
\hline $8 \mathrm{~d} \ldots \ldots \ldots \ldots \ldots \ldots$ & 11 & $\ldots$ & $\ldots$ & $\ldots$ & $\ldots$ & No emission & $\ldots$ \\
\hline $10 \mathrm{a} \ldots \ldots \ldots \ldots \ldots$ & 4 & $0.39 \pm 0.10$ & $0.76 \pm 0.17$ & 39.4: & -5 & dSy2 & -2.8 \\
\hline 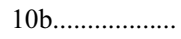 & 3 & $\ldots$ & $\ldots$ & $\ldots$ & $\ldots$ & No emission & 2.6 \\
\hline $10 \mathrm{c} \ldots \ldots \ldots \ldots \ldots$ & 3 & $-0.33 \pm 0.02$ & $\ldots$ & 39.6 & -15 & SFG? & -12.5 \\
\hline $10 \mathrm{~d} \ldots \ldots \ldots \ldots \ldots$ & 3 & $-0.29 \pm 0.03$ & $\ldots$ & 39.0 & -10 & SFG? & -9.4 \\
\hline $15 \mathrm{a} \ldots \ldots \ldots \ldots \ldots$ & 4 & $-0.01 \pm 0.08$ & $-0.19 \pm 0.15$ & 40.0 & -3 & dLINER & 0.3 \\
\hline 15b......................... & 4 & $\ldots$ & $\ldots$ & $\ldots$ & $\ldots$ & No emission & 4.4 \\
\hline $15 \mathrm{c} \ldots \ldots \ldots \ldots \ldots$ & 4 & $\ldots$ & $\ldots$ & $\ldots$ & $\ldots$ & No emission & 5.7 \\
\hline $15 \mathrm{~d} . \ldots \ldots \ldots \ldots \ldots \ldots$ & 4 & $-0.02 \pm 0.04$ & $0.11 \pm 0.06$ & 39.8 & -9 & LINER & -8.6 \\
\hline $30 \mathrm{a} \ldots \ldots \ldots \ldots \ldots \ldots$ & 3 & $\ldots$ & $\ldots$ & $\ldots$ & $\ldots$ & No emission & 3.8 \\
\hline 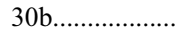 & 2 & $\ldots$ & $-0.34 \pm 0.16$ & $\ldots$ & $\ldots$ & LLAGN? & 2.4 \\
\hline +31a.................... & 3 & $-0.95 \pm 0.02$ & $0.44 \pm 0.02$ & 40.2 & -693 & H IIgal & -592.1 \\
\hline 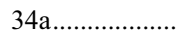 & 5 & $0.02 \pm 0.08$ & $\ldots$ & 39.8: & -3 & LLAGN? & 1.7 \\
\hline$+34 \mathrm{c} \ldots \ldots \ldots \ldots \ldots$ & 6 & $-0.52 \pm 0.05$ & $-0.02 \pm 0.06$ & 39.6 & -97 & SFG & -100.4 \\
\hline 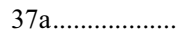 & 7 & $0.23 \pm 0.06$ & $0.22 \pm 0.22$ & 40.0: & -5 & dLINER & -2.9 \\
\hline 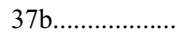 & 13 & $-0.19 \pm 0.07$ & $0.10 \pm 0.16$ & 39.7 & -5 & LINER & -0.6 \\
\hline $37 \mathrm{c} \ldots \ldots \ldots \ldots \ldots$ & 10 & $-0.28 \pm 0.10$ & $\ldots$ & 39.2: & -2 & LLAGN? & 0.3 \\
\hline 37d..................... & 6 & $-0.57 \pm 0.01$ & $-0.46 \pm 0.08$ & 40.1 & -44 & SFG & -42.2 \\
\hline $40 \mathrm{a} \ldots \ldots \ldots \ldots \ldots$ & 5 & $\ldots$ & $\ldots$ & $\ldots$ & $\ldots$ & No emission & 1.0 \\
\hline 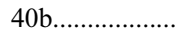 & 6 & $\ldots$ & $\ldots$ & $\ldots$ & $\ldots$ & No emission & 3.1 \\
\hline $40 \mathrm{c} \ldots \ldots \ldots \ldots \ldots \ldots$ & 8 & $-0.23 \pm 0.01$ & $-0.30 \pm 0.09$ & 40.2 & -14 & SFG & -12.0 \\
\hline $40 \mathrm{~d}$ & 5 & $-0.28 \pm 0.01$ & $-0.46 \pm 0.08$ & 40.8 & -38 & SFG & -40.1 \\
\hline $40 \mathrm{e} . \ldots \ldots \ldots \ldots \ldots . . . . . . . .$. & 6 & $-0.31 \pm 0.01$ & $\ldots$ & 39.4 & -6 & SFG? & -3.2 \\
\hline $54 \mathrm{a} \ldots \ldots \ldots \ldots \ldots \ldots$ & 1 & $-0.90 \pm 0.03$ & $0.51 \pm 0.06$ & 38.7 & -18 & H IIgal & -14.4 \\
\hline$+54 \mathrm{~b} \ldots \ldots \ldots \ldots \ldots$ & 1 & $-1.36 \pm 0.03$ & $0.68 \pm 0.03$ & 38.5 & -471 & H IIgal & -472.4 \\
\hline$+54 \mathrm{c} \ldots \ldots \ldots \ldots \ldots$ & 1 & $-1.12 \pm 0.02$ & $0.37 \pm 0.03$ & 37.8 & -84 & H IIgal & -78.5 \\
\hline$+54 \mathrm{~d} \ldots \ldots \ldots \ldots \ldots$ & 1 & $-1.23 \pm 0.05$ & $0.57 \pm 0.05$ & 37.8 & -102 & H IIgal & -102.7 \\
\hline $56 \mathrm{a} \ldots \ldots \ldots \ldots \ldots \ldots$ & 25 & $-0.42 \pm 0.01$ & $0.01 \pm 0.04$ & 40.4 & -20 & SFG & -16.8 \\
\hline$+56 \mathrm{~b} \ldots \ldots \ldots \ldots \ldots$ & 5 & $-0.04 \pm 0.01$ & $1.45 \pm 0.02$ & 39.9 & -52 & Sy2 & -52.1 \\
\hline $56 \mathrm{c} \ldots \ldots \ldots \ldots \ldots \ldots$ & 6 & $\ldots$ & $\ldots$ & $\ldots$ & $\ldots$ & No emission & 1.4 \\
\hline $56 \mathrm{~d} \ldots \ldots \ldots \ldots \ldots \ldots$ & 11 & $-0.28 \pm 0.02$ & $-0.10 \pm 0.02$ & 40.3 & -25 & SFG & -23.9 \\
\hline $56 \mathrm{e} . . . \ldots \ldots \ldots \ldots \ldots . . . . . . .$. & 7 & $-0.48 \pm 0.02$ & $\ldots$ & 39.9 & -14 & SFG? & -9.4 \\
\hline$+58 \mathrm{a} \ldots \ldots \ldots \ldots \ldots$ & 4 & $\ldots$ & $\ldots$ & 39.6 & -66 & Sy1.8 & -39.8 \\
\hline $58 \mathrm{~b} \ldots \ldots \ldots \ldots \ldots$ & 5 & $0.07 \pm 0.18$ & $\ldots$ & 39.8: & -3 & LLAGN? & 0.2 \\
\hline $58 \mathrm{c} \ldots \ldots \ldots \ldots \ldots \ldots$ & 5 & $0.10 \pm 0.11$ & $0.15 \pm 0.16$ & 39.7: & -6 & dLINER & -4.6 \\
\hline $58 \mathrm{~d} \ldots \ldots \ldots \ldots \ldots$ & 5 & $\ldots$ & $\ldots$ & $\ldots$ & $\ldots$ & No emission & 2.2 \\
\hline $58 \mathrm{e} \ldots \ldots \ldots \ldots \ldots \ldots$ & 5 & $-0.54 \pm 0.02$ & $-0.49 \pm 0.15$ & 40.0 & -28 & SFG & -22.4 \\
\hline $68 \mathrm{a} \ldots \ldots \ldots \ldots \ldots \ldots$ & 3 & $\ldots$ & $\ldots$ & $\ldots$ & $\ldots$ & No emission & 0.9 \\
\hline 68b....................... & 2 & $-0.19 \pm 0.2$ & $0.14 \pm 0.17$ & 39.4: & -4 & dLINER & 1.1 \\
\hline $68 \mathrm{c} \ldots \ldots \ldots \ldots \ldots$ & 2 & $-0.06 \pm 0.02$ & $-0.02 \pm 0.05$ & 39.8 & -31 & LINER & -29.6 \\
\hline 74a & 14 & $\ldots$ & $\cdots$ & $\ldots$ & $\ldots$ & No emission & 1.3 \\
\hline 74b....................... & 11 & $\ldots$ & $\ldots$ & $\ldots$ & $\ldots$ & No emission & 2.5 \\
\hline $74 \mathrm{c} \ldots \ldots \ldots \ldots \ldots$ & 7 & $\ldots$ & $\ldots$ & $\ldots$ & $\ldots$ & No emission & 2.8 \\
\hline 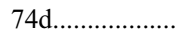 & 9 & $\ldots$ & $\ldots$ & $\ldots$ & $\ldots$ & No emission & 2.2 \\
\hline 79a.......................... & 4 & $-0.28 \pm 0.05$ & $0.28 \pm 0.11$ & 39.6 & -7 & LINER & -4.6 \\
\hline 79b....................... & 5 & $-0.31 \pm 0.05$ & $\ldots$ & 40.0 & -8 & SFG? & -6.0 \\
\hline 79c..................... & 3 & $\ldots$ & $\cdots$ & $\ldots$ & $\ldots$ & No emission & 5.0 \\
\hline$+79 \mathrm{~d} \ldots \ldots \ldots \ldots \ldots$ & 5 & $-1.05 \pm 0.05$ & $0.39 \pm 0.04$ & 38.3 & -47 & H IIgal & -34.6 \\
\hline 88b...................... & 3 & $0.28 \pm 0.09$ & $0.51 \pm 0.20$ & 39.4: & -7 & dSy2 & -6.1 \\
\hline $88 \mathrm{c} \ldots \ldots \ldots \ldots \ldots$ & 6 & $-0.50 \pm 0.01$ & $-0.41 \pm 0.04$ & 40.0 & -30 & SFG & -29.9 \\
\hline 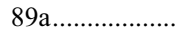 & 5 & $-0.40 \pm 0.02$ & $-0.37 \pm 0.07$ & 39.8 & -10 & SFG & -8.7 \\
\hline $89 \mathrm{~b} . \ldots \ldots \ldots \ldots \ldots \ldots$ & 6 & $-0.56 \pm 0.01$ & $-0.10 \pm 0.04$ & 40.3 & -39 & SFG & -31.3 \\
\hline 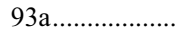 & 3 & $0.13 \pm 0.11$ & $0.24 \pm 0.14$ & 39.7 & -7 & dLINER & -5.4 \\
\hline 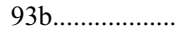 & 2 & $-0.39 \pm 0.02$ & $\ldots$ & 39.6 & -22 & SFG? & -16.4 \\
\hline $93 \mathrm{c} \ldots \ldots \ldots \ldots \ldots$ & 3 & $-0.09 \pm 0.10$ & $-0.12 \pm 0.17$ & 39.3 & -4 & dLINER & -1.8 \\
\hline $93 \mathrm{~d} . . . \ldots \ldots \ldots \ldots \ldots . . . . . .$. & 3 & $\ldots$ & $\ldots$ & $\ldots$ & $\ldots$ & No emission & 3.8 \\
\hline $94 \mathrm{a} \ldots \ldots \ldots \ldots \ldots$ & 6 & $\ldots$ & $\ldots$ & $\ldots$ & $\ldots$ & No emission & 4.5 \\
\hline 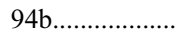 & 6 & $\ldots$ & $\ldots$ & $\ldots$ & $\ldots$ & No emission & 4.3 \\
\hline $94 \mathrm{c} \ldots \ldots \ldots \ldots \ldots \ldots$ & 7 & $\ldots$ & $\ldots$ & $\ldots$ & $\ldots$ & No emission & 6.1 \\
\hline 94d...................... & 6 & $\ldots$ & $\ldots$ & $\ldots$ & $\ldots$ & No emission & 6.0 \\
\hline $98 \mathrm{a} \ldots \ldots \ldots \ldots \ldots$ & 5 & $\ldots$ & $\ldots$ & $\ldots$ & $\ldots$ & No emission & 8.6 \\
\hline 98b..................... & 4 & $\ldots$ & $\ldots$ & $\ldots$ & $\ldots$ & No emission & 3.0 \\
\hline
\end{tabular}




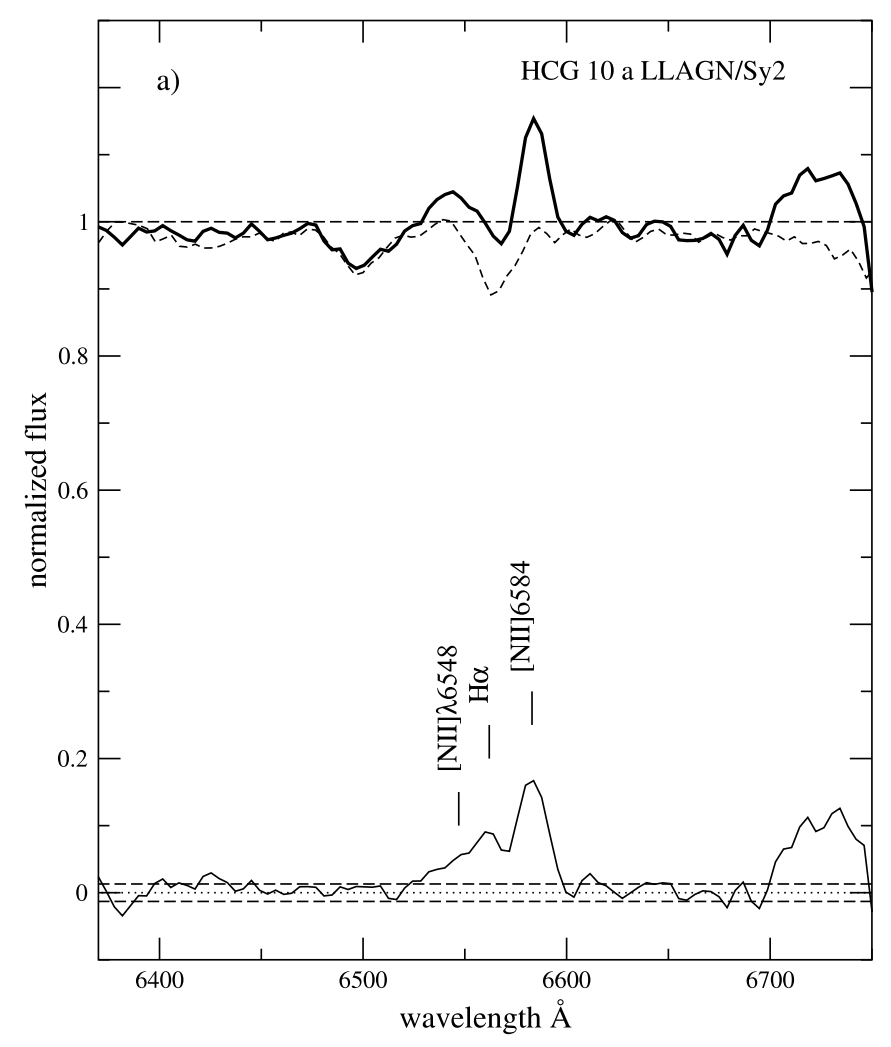

FIG. $1 a$

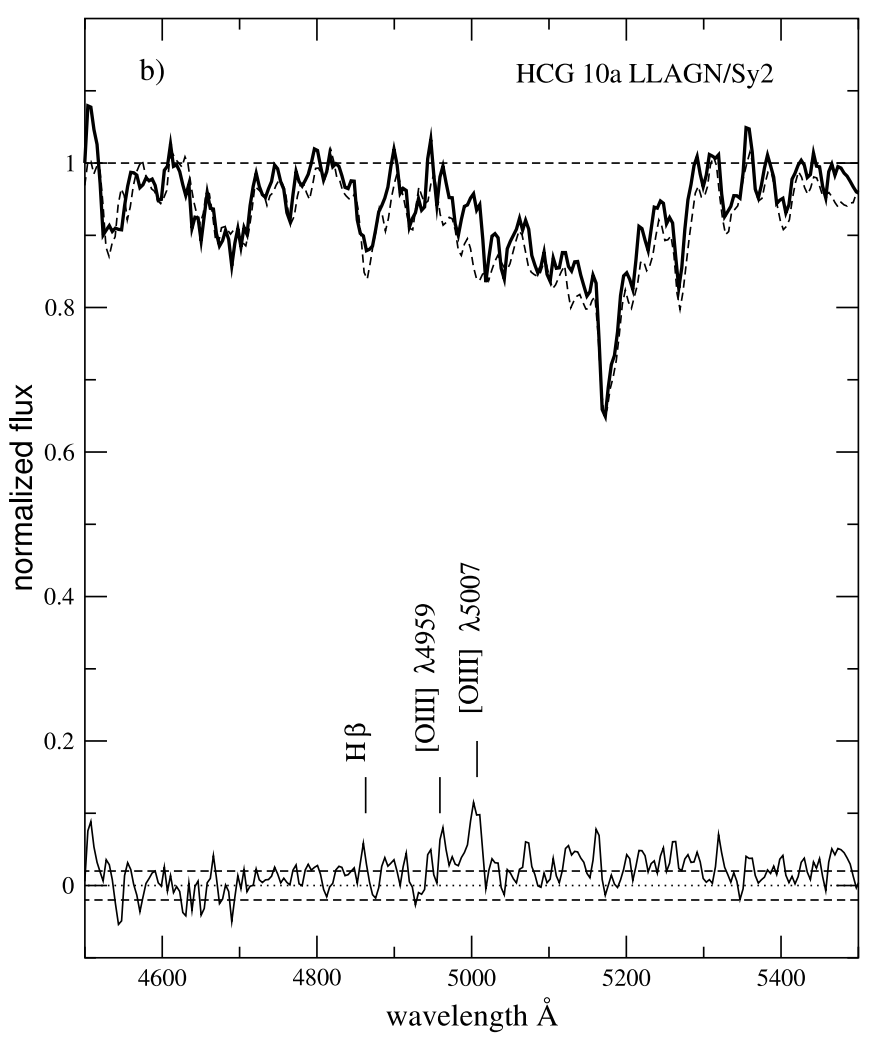

FIG. $1 b$

FIG. 1.-Examples of template subtraction in $(a)$ the $\mathrm{H} \alpha$ and $(b)$ the $\mathrm{H} \beta$ regions. The galaxy is the LLAGN candidate HCG10a. The top of the figures shows the

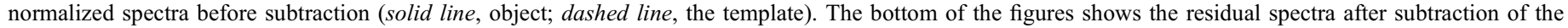
template, with the zero level (dotted line) and $\pm 1 \sigma$ rms (dashed line) indicated. 


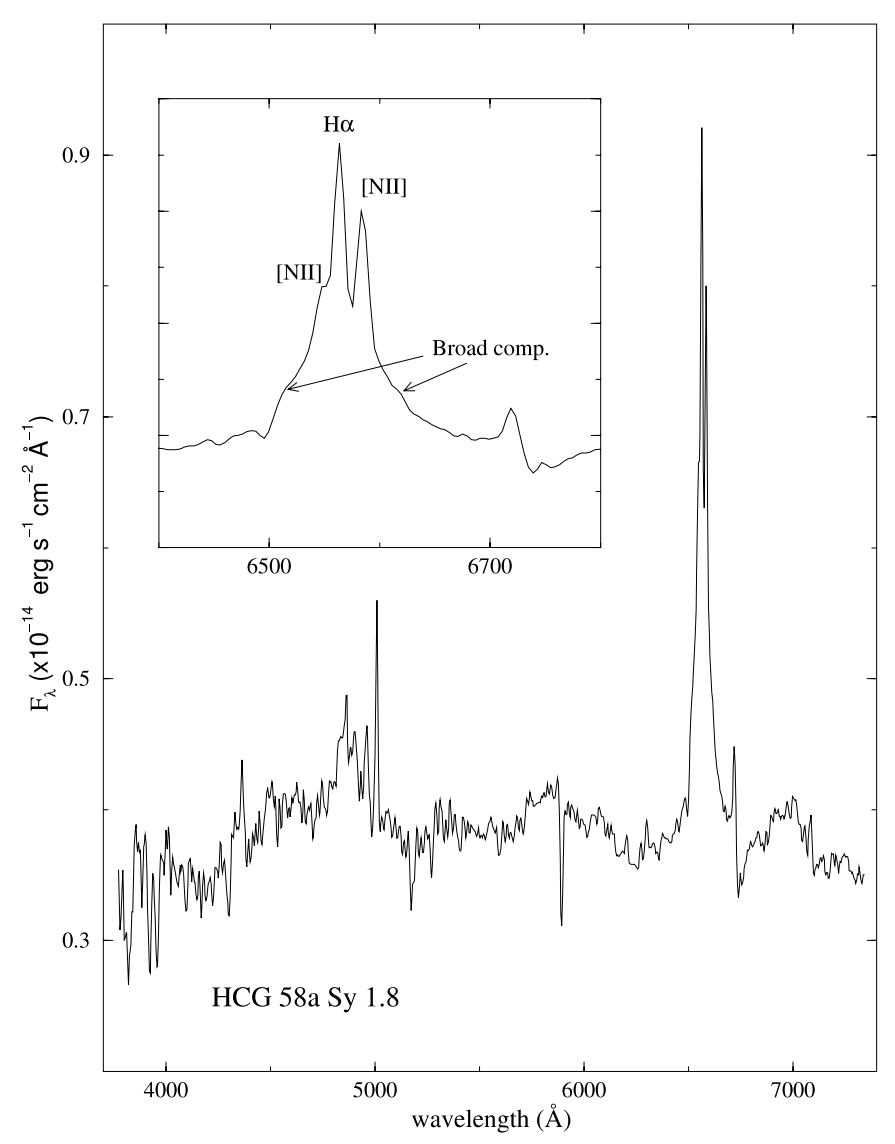

Fig. 2.-Optical spectrum of HCG 58a presented in its rest frame. This galaxy looks like a Seyfert 2, but the presence of large wings around $\mathrm{H} \beta$ (already evident in the spectrum) and $\mathrm{H} \alpha$ (evident in the enlarged section) make it a Seyfert 1.8.

the luminosity is of the order of $10 \%$. Luminosities with uncertainties larger than $20 \%$ are identified by a colon (uncertainties are always smaller than $30 \%$ ). The uncertainty on the $\mathrm{EW}$, which is mainly due to the template subtraction, is of the order of $1 \AA$ A.

\subsection{Activity Classification}

Figure 2 shows the final spectrum for the galaxy HCG 58a, which is the most active galaxy in our sample. As can be seen in the enlarged region, the presence of weak, broad components around $\mathrm{H} \alpha$ (also visible around $\mathrm{H} \beta$ ) suggests this is a Seyfert 1.8 (Osterbrock 1981). We emphasize that HCG 58a is the first Seyfert 1 galaxy (although of an intermediate type) that we have found thus far, after classifying 118 galaxies in 34 HCGs. This result confirms the rarity of luminous AGNs in CGs (Coziol et al. 1998b, 2000).

The standard diagnostic diagram (Veilleux \& Osterbrock 1987) for the emission-line galaxies in our sample is shown in Figure 3 (the Seyfert 1.8 is not included). It suggests that the LLAGNs are either LINER or Seyfert 2. The spectra of these galaxies are shown in Figures $4 a$ and $4 b$. For comparison, we also show in Figure $5 a$ the four LLAGN candidates that we were not able to classify. The similarities in the spectra are obvious. Note in particular the weakness of the $\mathrm{H} \alpha$ line in these galaxies, which prompts us to classify them as possible AGNs. In contrast, we show in Figure $5 b$ the spectra of those galaxies that show weak star formation activity (star-forming galaxies or SFGs). We see that the $\mathrm{H} \alpha$ line is stronger than the

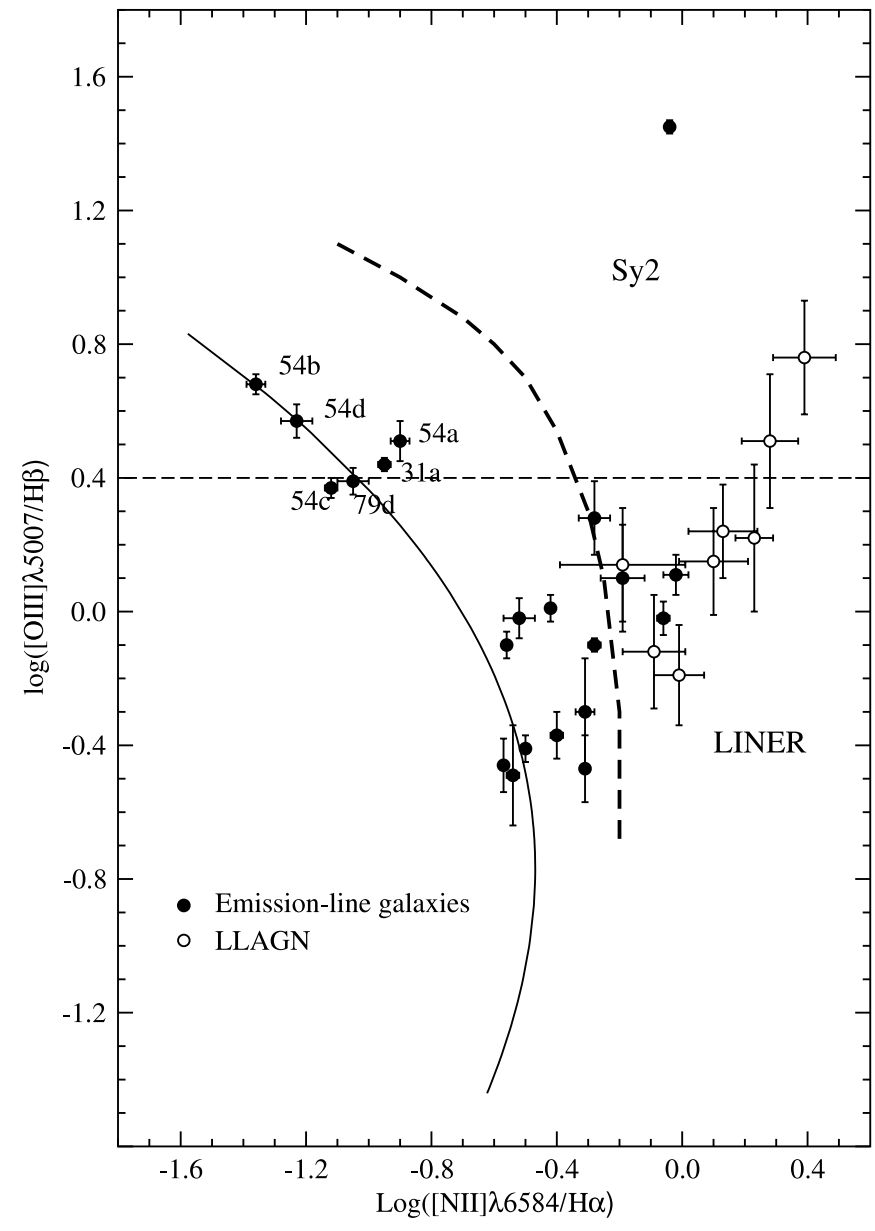

FIG. 3.-Standard diagnostic diagram for the emission-line galaxies in our sample. The dashed curve separates thermal (left) from nonthermal (right) galaxies, while the continuous curve is the locus traced by normal $\mathrm{H}$ II regions. The horizontal dashed line separates low-excitation, high-metallicity starforming galaxies (bottom) from high-excitation, low-metallicity star-forming galaxies (top).

two nitrogen lines in these galaxies, which is a clear indication for star formation. Another feature in common with luminous SFGs is their late-type morphology (see the morphological classification in Table 1, col. [7]). The two exceptions are HCG 56e and 79b.

The activity type adopted for each galaxy is reported in Table 2 (col. [7]). They are identified as: quiescent galaxy (No emission), star-forming galaxy (SFG) or $\mathrm{H}$ II galaxies (highexcitation, low-metallicity SFG), LINER, Seyfert 2 (Sy2), Seyfert 1 (Sy1), and LLAGNs (dSy2 and dLINER). A question mark after the activity type indicates some uncertainty because only one emission line complex (either around $\mathrm{H} \alpha$ or $\mathrm{H} \beta$ ) is detected after template subtraction. This was the case for 10 galaxies, which we tentatively classified four LLAGN candidates and six weak SFGs.

Because of the difference in morphology between the templates used and the emission-line galaxies, we expect our line ratios to be somewhat underestimated. However, we note that to change the nature of the LLAGN from LINER to starforming galaxies in the diagnostic diagram, we would need to have underestimated the $\mathrm{H} \alpha$ fluxes by a factor of more than 20, which seems unreasonably large (see Miller \& Owen 2002 for a similar result in clusters of galaxies).

In Figure 6, we compare the $\mathrm{H} \alpha$ luminosity of the emissionline galaxies with the equivalent width of the $\mathrm{H} \alpha+\left[\mathrm{N}_{\mathrm{II}}\right]$ 

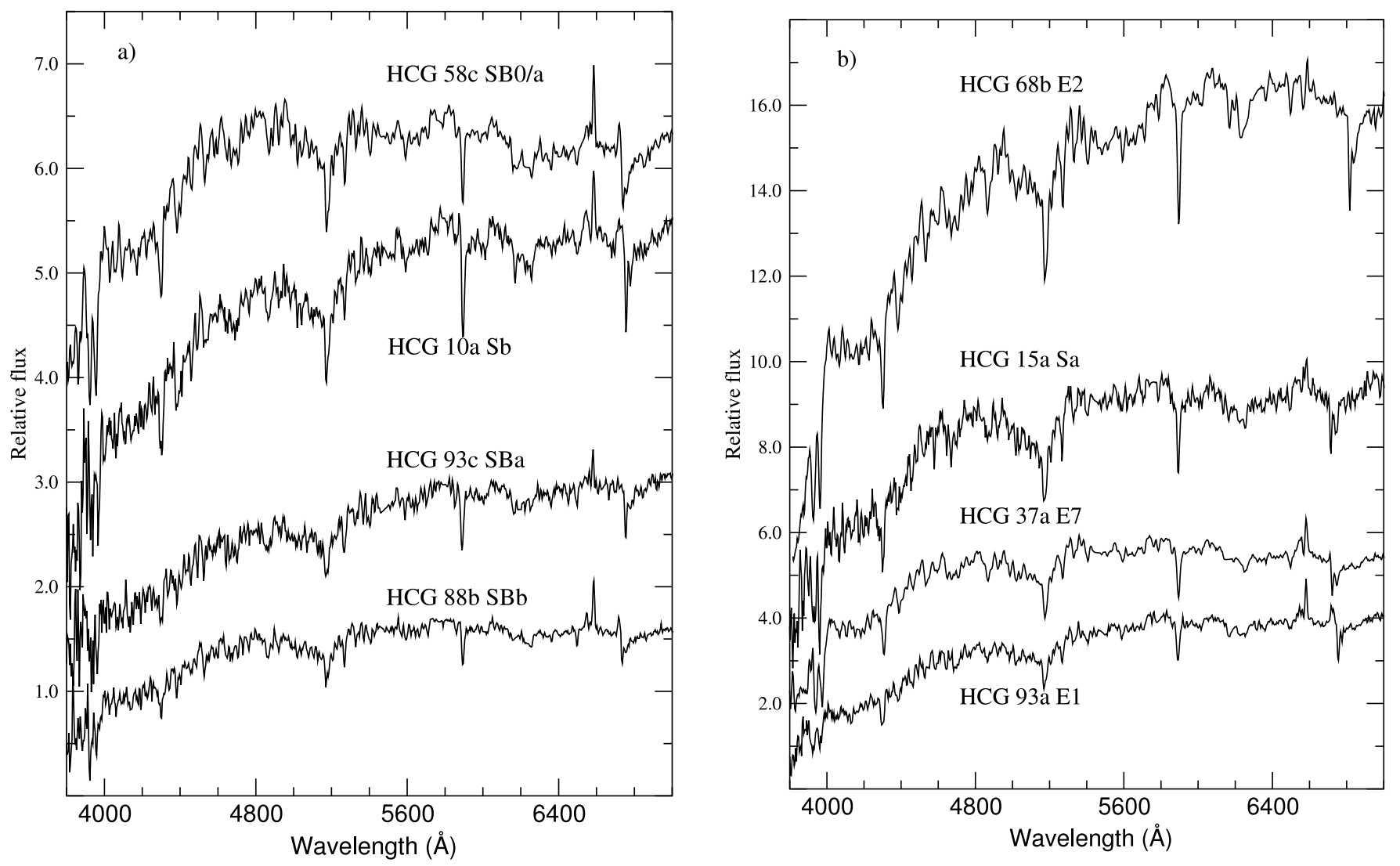

FIG. 4.- Spectra of the confirmed LLAGNs in our sample. All spectra are in the rest frame and slightly shifted in flux to facilitate comparison. Note the weakness of the $\mathrm{H} \alpha$ line in emission compared with the two [N II] lines. 

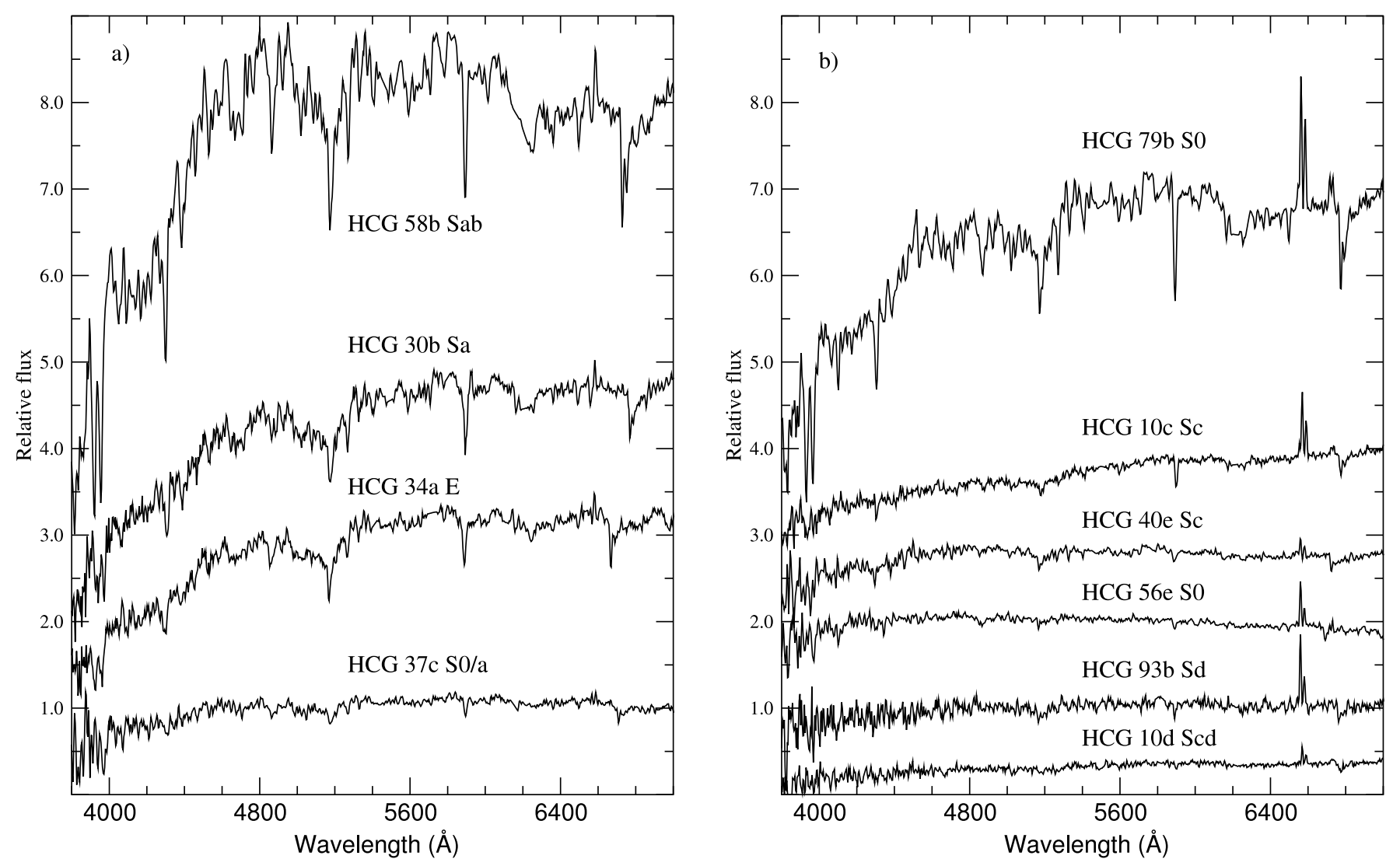

Fig. 5.-Spectra of $(a)$ the LLAGN and $(b)$ the SFG candidates. All spectra are reduced to the rest frame and slightly shifted in flux to facilitate comparison. The LLAGN candidates share with the confirmed LLAGNs displayed in Fig. 4 an early-type morphology of the host galaxies. The SFG candidates, with the exception of HCG $79 \mathrm{~b}$ and 56e, are all late-type spirals. 


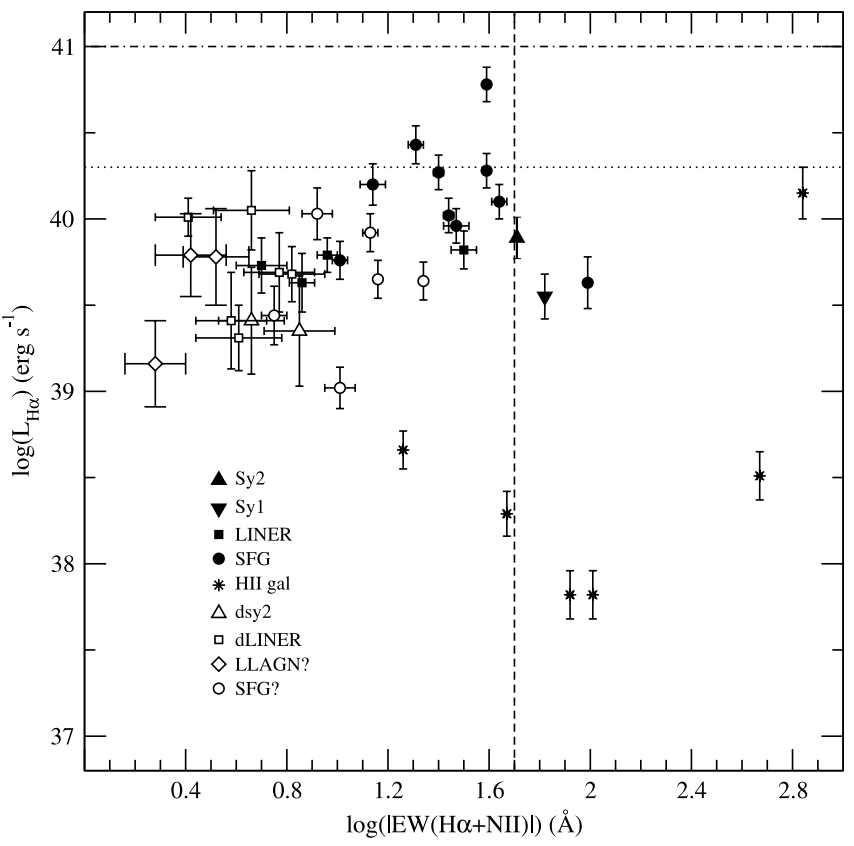

FIG. 6. $-\mathrm{H} \alpha$ luminosity of the emission line galaxies (after template subtraction) as a function of the $\mathrm{EW}$ of the $\left(\mathrm{H} \alpha+\left[\mathrm{N}_{\mathrm{II}}\right]\right)$ lines. The two horizontal lines are the mean luminosity for Markarian starburst galaxies (dash-dotted line) and their lower limit (dotted line). The vertical (dashed) line marks the EW lower limit for actively star-forming galaxies.

lines. The horizontal lines indicate the mean $\mathrm{H} \alpha$ luminosity (dot-dashed line) and lower limits as observed in nuclear starburst galaxies (Contini et al. 1998). The vertical (dashed line) is the dividing line between galaxies with weak and active star formation (Kennicutt \& Kent 1983). It can be seen that very few SFGs in our sample qualify as a starburst or an actively star-forming galaxy. In Figure 6, one can see that what seems to distinguish most LINERS and LLAGNs from SFGs are their slightly lower luminosity and distinctly smaller EW. The difference in EW, in particular, is important, since it suggests the continuum in these galaxies has a different origin (by definition, the EW is the ratio of the flux in the emission line to the continuum). This difference could be a sign of a weak AGN or of a significantly different ionizing stellar population.

Although we believe our classification is fair, we remind the reader that there is an ongoing discussion about the AGN nature of LLAGNs and LINERs (see the reviews by Lawrence 1999 and Ho 1999). Obviously, more observations will be needed before we can understand what the role of star formation and AGN activity is in these galaxies (see, for example, Turner et al. 2001).

In Table 3, we compare the distribution of type of activity in the present sample with that obtained in our previous analyses (Coziol et al. 1998b, 2000). For comparison, the luminous
LINER and Seyfert galaxies were included in the same AGN bin. The distribution of activity type in our new sample is fully consistent with what we observed before.

\subsection{Activity Index}

In order to quantify the level of activity in the groups, we define a new spectroscopic index for the galaxies. Since we want this index to be sensitive to the different stellar populations, and not only the ionized gas, we use the equivalent width as measured in a window centered on $\mathrm{H} \alpha$ (Copetti et al. 1986; Coziol 1996). The size of the window was chosen by considering the typical EWs of $\mathrm{H} \alpha$ lines (in absorption) in the spectra of A stars, which are known to dominate the stellar population after most of the OB stars have disappeared (as in poststarburst or gas-stripped galaxies; see Rose 1985; Leonardi \& Rose 1996; Barbaro \& Poggianti 1997) and which are responsible for the strong absorption features observed in LLAGNs (Coziol et al. 1998b). We define the activity index, $\mathrm{EW}_{\mathrm{act}}$, as the equivalent width measured in the rest frame of the galaxy between 6500 and $6600 \AA$ in the original spectra (i.e., without template subtraction). Defined in this way, the activity index covers all the possible active states of a galaxy: active (star formation and/or AGN), decreasing star formation (poststarburst or gas stripping), and quiescent (intermediate and old stellar populations), and has the advantage that it can be easily measured in all the galaxies. The value of the activity index as measured in each galaxy in our sample is given in the last column of Table 2 . The uncertainty in this value is of the order of $\pm 0.2 \AA$. It was estimated using the mean of the EW of all the structures (real or spurious) encountered in the continuum around the window in the four template galaxies. Note that, because of their redshifts, the $\mathrm{O}_{2}$ atmospheric band at $6870 \AA$ fell right within the window of the galaxies in HCG 8, preventing us from measuring an $\mathrm{EW}_{\mathrm{act}}$.

In order to increase our sample of galaxies in CGs, we have measured the activity index in those galaxies that were observed in our first spectroscopic analysis of HCGs (Coziol et al. 1998b). Only four galaxies from this previous article are also found in the present study: HCG 40a, b, e, and HCG $88 \mathrm{~b}$. The activity indices found for these galaxies based on the $4 \mathrm{~m}$ spectra are $0.9,3.6,-3.0$, and $-5.0 \AA$, respectively. This compares very well with the activity indices measured in our new spectra: $1.0,3.1,-3.2$, and $-6.1 \AA$, respectively.

Not considering two groups that we reobserved in the present study and five groups in which only one galaxy of each group was observed, we can add 10 CGs to our sample. The results are presented in Table 4, together with the morphological type and activity classification for each galaxy.

\section{ANALYSIS}

\subsection{Utility and Robustness of the Activity Index}

Ideally, by using the mean of the activity index we should be able to discriminate between active and nonactive CGs. By

TABLE 3

Activity Classification in CGs

\begin{tabular}{|c|c|c|c|c|}
\hline Sample & $\begin{array}{c}\text { No Emission } \\
(\%)\end{array}$ & $\begin{array}{c}\text { LLAGN } \\
(\%)\end{array}$ & $\begin{array}{c}\text { AGN } \\
(\%)\end{array}$ & $\begin{array}{l}\text { SFG } \\
(\%)\end{array}$ \\
\hline This work (65 galaxies/19 groups) .................... & 38 & 19 & 9 & 34 \\
\hline 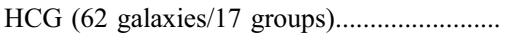 & 40 & 21 & 18 & 21 \\
\hline SCG (193 galaxies/49 groups) …...................... & 27 & 22 & 19 & 32 \\
\hline
\end{tabular}


TABLE 4

Activity Indices and Characteristics of the Additional Sample

\begin{tabular}{|c|c|c|c|c|}
\hline $\mathrm{HCG}$ & Activity Type ${ }^{a}$ & Morphology $\mathrm{y}^{\mathrm{b}}$ & $T$ & $\begin{array}{c}\mathrm{EW}_{\text {act }} \\
(\AA)\end{array}$ \\
\hline $4 \mathrm{a} \ldots \ldots \ldots \ldots \ldots \ldots \ldots$ & SFG & $\mathrm{Sc}$ & 5 & -127.0 \\
\hline 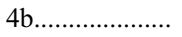 & SFG & $\mathrm{Sc}$ & 5 & -431.8 \\
\hline ............. & SFG & E4 & -5 & -38.4 \\
\hline $16 \mathrm{a} . . . \ldots \ldots \ldots \ldots \ldots$ & LINER & SBab & 2 & -8.2 \\
\hline $16 \mathrm{~b} \ldots \ldots \ldots \ldots \ldots \ldots$ & Sy2 & $\mathrm{Sab}$ & 2 & -12.5 \\
\hline $16 \mathrm{c} \ldots \ldots \ldots \ldots \ldots \ldots$ & SFG & $\operatorname{Im}$ & 10 & -180.2 \\
\hline $16 \mathrm{~d} \ldots \ldots$ & SFG & $\operatorname{Im}$ & 10 & -63.1 \\
\hline $22 \mathrm{a} \ldots \ldots \ldots \ldots \ldots . . . . . . . .$. & LLAGN & E2 & -5 & 0.8 \\
\hline $22 b \ldots \ldots$ & No emission & $\mathrm{Sa}$ & 1 & 5.1 \\
\hline $22 \mathrm{c} \ldots \ldots$ & SFG & SBcd & 6 & -0.4 \\
\hline $23 \mathrm{a} \ldots \ldots \ldots \ldots \ldots$ & LLAGN & $\mathrm{Sab}$ & 2 & -0.1 \\
\hline $23 \mathrm{c} \ldots \ldots \ldots \ldots \ldots \ldots$ & LLAGN & S0 & -2 & 0.4 \\
\hline $23 \mathrm{~d} \ldots \ldots$ & SFG & $\mathrm{Sd}$ & 7 & -219.8 \\
\hline $42 \mathrm{a} \ldots \ldots \ldots \ldots \ldots$ & LLAGN & E3 & -5 & -1.0 \\
\hline $42 b \ldots \ldots$ & No emission & SB0 & -2 & 2.8 \\
\hline $42 \mathrm{c} \ldots \ldots$ & No emission & E2 & -5 & 3.1 \\
\hline $62 \mathrm{a} \ldots \ldots \ldots \ldots \ldots \ldots$ & LLAGN & E3 & -5 & -2.5 \\
\hline $62 b \ldots \ldots$ & No emission & S0 & -2 & 3.5 \\
\hline $62 \mathrm{c} \ldots \ldots \ldots \ldots \ldots$ & No emission & S0 & -2 & 3.1 \\
\hline 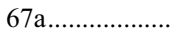 & No emission & E1 & -5 & 3.6 \\
\hline $67 \mathrm{~b} \ldots \ldots$ & SFG & $\mathrm{Sc}$ & 5 & -19.0 \\
\hline $86 \mathrm{a} \ldots \ldots \ldots \ldots \ldots \ldots$ & LLAGN & E2 & -5 & 1.2 \\
\hline $86 \mathrm{~b} \ldots \ldots \ldots \ldots \ldots \ldots$ & LLAGN & E2 & -5 & -1.2 \\
\hline $86 \mathrm{c} \ldots \ldots \ldots \ldots \ldots . . . . . . .$. & LINER & SB0 & -2 & -2.7 \\
\hline $86 \mathrm{~d} \ldots .$. & No emission & S0 & -2 & 2.7 \\
\hline $87 \mathrm{a} \ldots \ldots \ldots \ldots \ldots . . . . . . .$. & LLAGN & $\mathrm{Sbc}$ & 4 & 0.1 \\
\hline 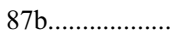 & LLAGN & S0 & -2 & 1.6 \\
\hline $90 \mathrm{a} . . . \ldots \ldots \ldots \ldots \ldots$ & Sy2 & $\mathrm{Sa}$ & 1 & -3.4 \\
\hline 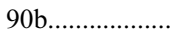 & LINER & E0 & -2 & -10.1 \\
\hline $90 \mathrm{c} \ldots \ldots \ldots \ldots \ldots . . . . . . .$. & No emission & E0 & -2 & 2.2 \\
\hline 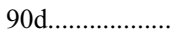 & LINER & $\operatorname{Im}$ & 10 & -8.4 \\
\hline
\end{tabular}

a Coziol et al. (1998b).

b Hickson (1982).

active CGs we mean those groups that harbor star forming galaxies and/or AGNs. To verify this, we compare in Figure $7 a$ the activity index as measured in galaxies with different activity types. Since the range of the index is large, we take the logarithm and discriminate between absorption $\left(\mathrm{EW}_{\mathrm{act}}>0\right)$ and emission $\left(\mathrm{EW}_{\mathrm{act}}<0\right)$ using different symbols. In general, SFGs and luminous AGNs (Sy1, Sy2, and LINER) have $\mathrm{EW}_{\text {act }}<-6$ (or $\log \left|\mathrm{EW}_{\mathrm{act}}\right|>0.8$ ). This property allows us to separate them from quiescent galaxies and LLAGNs. We therefore adopt a mean of $\mathrm{EW}_{\text {act }}<-6$ to separate active from inactive groups. Note that for a mean $\mathrm{EW}_{\text {act }}>-6$, a negative mean index would indicate some activity but at a significantly lower level than for active groups.

In Coziol et al. (1998b), the type of activity presented by the different galaxies was found to be correlated with their morphology. To verify if this phenomenon is also observed in the present sample, we compare the activity index with the morphological type of the galaxies in Figure $7 b$. In general, we find the SFGs in late-type $(T>3)$ galaxies and the quiescent galaxies in early-type $(T<1)$. The exceptions are HCG 56d (S0) and 40d (SBa) for the SFGs and HCG 30a (SBc) for the quiescent galaxies. We also note that AGNs and LLAGNs are usually found in intermediate and early-type galaxies.

In Figure 8, we repeat our analysis for the galaxies in the complementary sample studied by Coziol et al. (1998a, 1998b). We distinguish the same trends as observed in our
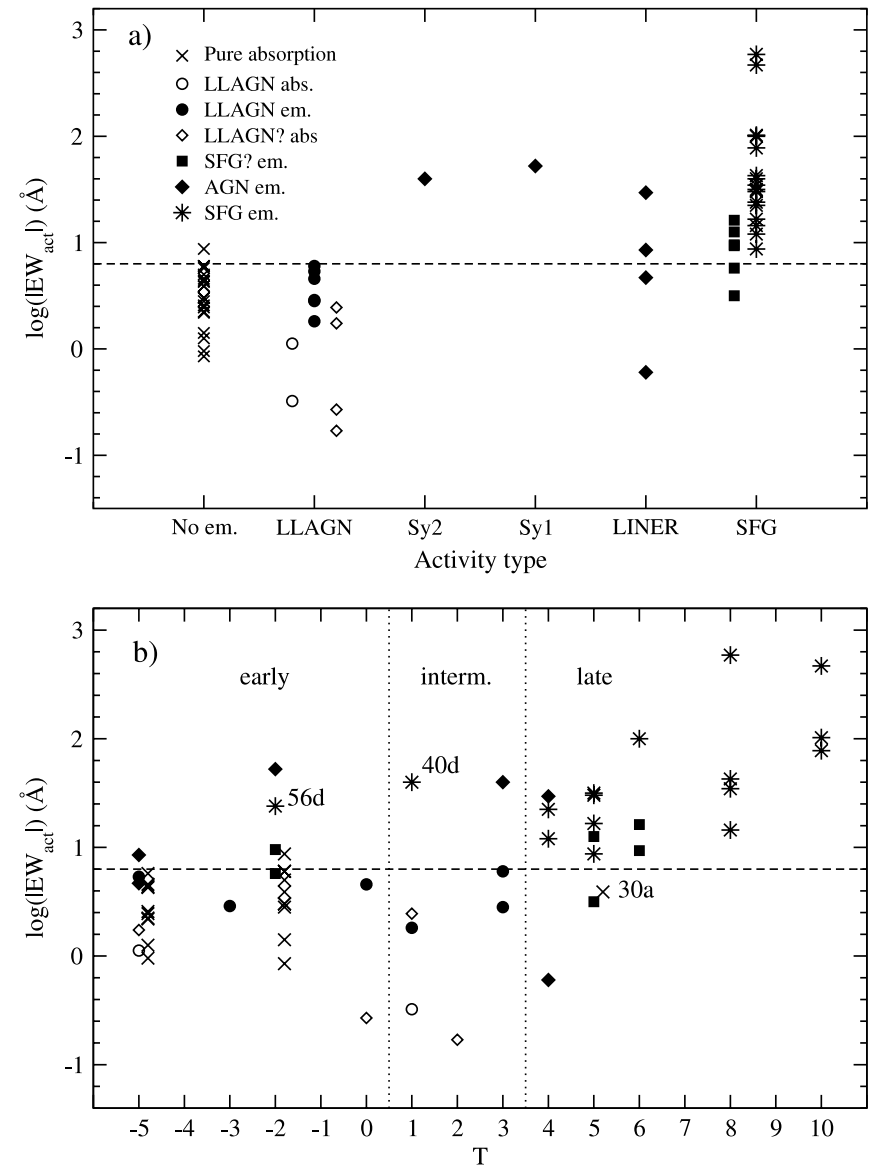

FIG. 7.- Value of the activity index in galaxies with (a) different activity types and $(b)$ different morphological types . The horizontal dashed line separates SFGs from quiescent galaxies and LLAGNs. Using different symbols, we also distinguish between absorption $\left(\mathrm{EW}_{\mathrm{act}}>0\right)$ and emission $\left(\mathrm{EW}_{\mathrm{act}}<0\right)$. The vertical (dotted) lines separate the morphology axis into three categories: early-, intermediate-, and late-type.

new sample. This result suggests that the activity index is independent of the type of telescope and reduction aperture used. This is most readily attributed to the compact nature of the light and ionized gas distributions in these galaxies (see our comment in $\S 3.1$ ).

\subsection{Definition of Evolutionary Levels for CGs}

As noted previously in Coziol et al. (1998b), the relation between morphology and activity as found in CGs is not specific to these systems but is general to all galaxies independent of their environment (see, e.g., Kennicutt 1992). The same study showed that taken as a group, however, the morphology-activity relation was correlated with the density of the groups. This suggests that we should combine the mean activity index with the mean morphological type of galaxies in a CG in order to quantify their evolutionary state. If activity is a consequence of galaxy interactions and if interactions lead to the formation of early-type systems, we would thus expect "evolved" groups to be inactive and rich in early-type galaxies, whereas "unevolved" groups would be active and rich in late-type galaxies. Another possibility is for groups to be unusually active (or inactive) considering the morphologies of their members. In the interaction scenario, such unusually active groups may constitute a genuine intermediate evolutionary level. 

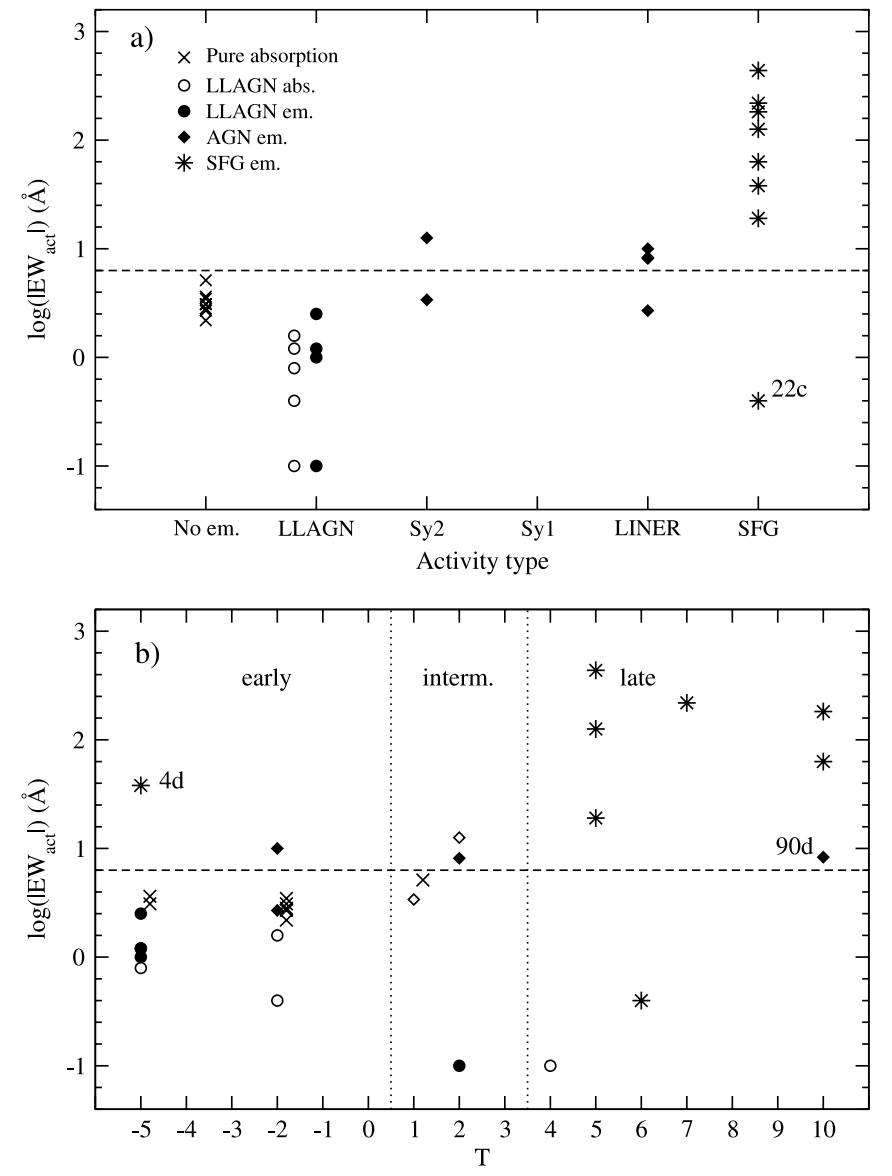

Fig. 8. - Value of the activity index as a function of $(a)$ activity types and (b) morphology for the galaxies in the additional sample. The meaning of the symbols is the same as in Fig. 7.

The mean morphological types and mean activity indices of the 27 CGs in our joined samples are reported in Table 5 (cols. [10] and [11], respectively). Note that since only one member galaxy was observed for HCG 31, this group was omitted from the rest of our analysis. In this table we have also collected other useful information on the groups as found in the literature: the systemic redshifts (col. [2]), as listed by Hickson in catalog VII/213 (at the CDS); a flag indicating diffuse X-ray emission (col. [3]), as determined by Ponman et al. (1996); the number of galaxies for which we have a spectral classification $\left(N_{S}\right.$; col. [4]); the number of real members $\left(N_{\mathrm{H}}\right.$; col. [5]), as determined by Hickson (1982); the number of possible members $\left(N_{\mathrm{R}}\right.$; col. [6]), as determined by Ribeiro et al. (1998); and the deprojected velocity dispersions (col. [7]), projected radii (col. [8]), and adopted masses (col. [9]) for the groups, as listed by Hickson.

\subsection{Effect of Incompleteness on Our Analysis}

As mentioned in $\S 2$, our information on the activity of the groups is not complete because of observational constraints. To establish how this incompleteness might affect our analysis, we have assigned an activity type to the missing galaxies based on the trends between morphology and activity observed in Figures 7 and 8 and used this prediction to estimate the changes their incorporation could induce on the mean activity index and mean morphology in each group. In Table 6, we list the 16 groups for which the activity information is incomplete, identifying the missing galaxies and their morphology. In column (4) we report the observed mean activity index, and in column (5) we note the change expected: a plus sign indicates a decrease in activity, a minus sign indicates an increase in activity, and a 0 indicates no variation. It can be seen that including the missing galaxies would either have no impact or reinforce our analysis. The last two columns list both the actual mean morphological type as well as its value after taking into account the missing galaxies. Once again, only marginal changes are seen. This test suggests that our present analysis is not compromised by the incompleteness of our sample.

\subsection{Evolutionary Sequence and Spatial Configuration of CGS}

In Figure 9, we compare the mean level of activity with the mean morphological type of the galaxies in 27 HCGs. The symbols distinguish between active $\left(\log \left|\mathrm{EW}_{\text {act }}\right|>0.8\right)$ and inactive groups. Our sample of CGs clearly spans an evolutionary sequence. We see evolved CGs, which are inactive and dominated by elliptical galaxies, slightly less-evolved groups, which look unusually active considering the early-type morphologies of their members, and nonevolved groups, which are active and dominated by late-type spirals and irregular galaxies.

At this point, it is instructive to inspect the spatial (projected) configurations of the groups. Indeed, we find that groups that share the same evolutioary state also share the same configuration. We identify three main configuration types. Type A CGs are dominated by late-type spirals, and all show a loose configuration. The mean activity index for this type is high. The prototypes are HCG 16, 23, 88, and 89 and are shown in Figure $10 .^{2}$ HCG 30 and 87 may also be classified as type A, based on their spatial configuration, even though they are inactive. Type B consists of galaxies in apparent close contact, suggesting ongoing mergers. The prototypes are HCG 40, 56, 68, and 79 (Fig. 11). A similar configuration is found for HCG 34, 37, and 67. These are all unusually active groups, considering the morphologies of their members. The three groups HCG 22, 90, and 98 may also be classified as type B, even though they are inactive. Finally, type $\mathrm{C}$ is formed by CGs that are dominated by elliptical galaxies and are inactive. The configuration is loose, and the groups are dominated either by one giant elliptical, as in $\mathrm{HCG}$ 42, 62, 74, and 94 (Fig. 12), or by more than one elliptical galaxy, as in HCG 15 and 86 (Fig. 13).

Although HCG 10,58, and 93 seem to share the same evolutionary state as groups in type B, their spatial configurations are more similar to groups in type A and consequently defy any classification.

HCG 54 also seems somewhat special. This group, which is remarkably active, shows the same merging configuration as CGs of type B but with the important difference that it is formed by irregular galaxies. The diagnostic diagram (Fig. 3) suggests that the galaxies in this group are low-mass, lowmetallicity $\mathrm{H}$ II galaxies. This is in contrast with the other CGs in our sample, which are usually formed by massive, and probably metal-rich, galaxies (as judged from their positions in the diagnostic diagram). It is not certain, therefore, that HCG 54 describes the same phenomenon as the other groups.

\footnotetext{
${ }^{2}$ The finding charts shown in this article were obtained using SkyCat (http://archive.eso.org/skycat), which is a tool developed by ESO's Data Management and Very Large Telescope Project divisions with contributions from the Canadian Astronomical Data Centre.
} 
TABLE 5

Mean Activity Index and Morphology of CGs

\begin{tabular}{|c|c|c|c|c|c|c|c|c|c|c|}
\hline $\begin{array}{l}\mathrm{HCG} \\
(1)\end{array}$ & $\begin{array}{l}z^{\mathrm{a}} \\
(2)\end{array}$ & $\begin{array}{c}\text { Diffuse X-rays }{ }^{b} \\
\text { (3) }\end{array}$ & $\begin{array}{l}N_{S} \\
(4)\end{array}$ & $\begin{array}{l}N_{\mathrm{H}}^{\mathrm{a}} \\
(5)\end{array}$ & $\begin{array}{l}N_{\mathrm{R}}^{\mathrm{c}} \\
(6)\end{array}$ & $\begin{array}{l}\text { Velocity Dispersion }{ }^{\mathrm{a}} \\
\left(\mathrm{km} \mathrm{s}^{-1}\right) \\
(7)\end{array}$ & $\begin{array}{c}\text { Radius }^{\mathrm{a}} \\
(\mathrm{kpc}) \\
(8)\end{array}$ & $\begin{array}{c}\log (\text { mass })^{a} \\
(\mathrm{~g}) \\
(9)\end{array}$ & $\begin{array}{l}\langle T\rangle \\
(10)\end{array}$ & $\begin{array}{c}\left\langle\mathrm{EW}_{\mathrm{act}}\right\rangle \\
(11)\end{array}$ \\
\hline $04 \ldots \ldots \ldots \ldots \ldots \ldots \ldots$ & 0.0280 & $?$ & 3 & $\ldots$ & $\ldots$ & 575 & 58 & 46.45 & 1.7 & -199 \\
\hline $10 \ldots \ldots \ldots \ldots \ldots \ldots \ldots \ldots \ldots \ldots \ldots$ & 0.0161 & $\ldots$ & 4 & $\ldots$ & $\ldots$ & 363 & 93 & 46.09 & 2.2 & -4 \\
\hline $15 \ldots \ldots \ldots \ldots \ldots \ldots \ldots$ & 0.0228 & yes & 4 & 6 & $\ldots$ & 724 & 78 & 46.86 & -3.5 & 0 \\
\hline $16 \ldots \ldots \ldots \ldots \ldots \ldots \ldots$ & 0.0132 & yes & 4 & $\ldots$ & 7 & 204 & 45 & 45.41 & 6.0 & -66 \\
\hline $22 \ldots \ldots \ldots \ldots \ldots \ldots \ldots \ldots$ & 0.0090 & $\ldots$ & 3 & $\ldots$ & 4 & 18 & 27 & 43.70 & 0.7 & 2 \\
\hline $23 \ldots \ldots \ldots \ldots \ldots \ldots \ldots$ & 0.0161 & $\ldots$ & 3 & 4 & 8 & 275 & 66 & 45.95 & 2.3 & -73 \\
\hline $30 \ldots \ldots \ldots \ldots \ldots \ldots \ldots$ & 0.0154 & $\ldots$ & 2 & 4 & $\ldots$ & 110 & 51 & 44.96 & 3.0 & 3 \\
\hline 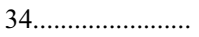 & 0.0307 & $\ldots$ & 2 & 4 & $\ldots$ & 550 & 15 & 45.90 & 0.5 & -49 \\
\hline ................ & 0.0223 & yes & 4 & 5 & $\ldots$ & 692 & 29 & 46.25 & 2.2 & -11 \\
\hline $40 \ldots \ldots \ldots \ldots \ldots \ldots$ & 0.0223 & $\ldots$ & 5 & 5 & 7 & 251 & 15 & 45.26 & 0.6 & -10 \\
\hline $42 \ldots \ldots \ldots \ldots \ldots \ldots \ldots$ & 0.0133 & yes & 3 & 4 & 11 & 363 & 45 & 45.83 & -4.0 & 2 \\
\hline ................ & 0.0049 & $\ldots$ & 4 & $\ldots$ & $\ldots$ & 182 & 2 & 43.88 & 9.5 & -167 \\
\hline $56 \ldots \ldots \ldots \ldots \ldots \ldots \ldots$ & 0.0270 & $\ldots$ & 5 & $\ldots$ & $\ldots$ & 282 & 21 & 45.34 & -0.6 & -20 \\
\hline $58 \ldots \ldots \ldots \ldots \ldots \ldots \ldots$ & 0.0207 & yes & 5 & $\ldots$ & $\ldots$ & 275 & 89 & 45.98 & 0.8 & -13 \\
\hline $62 \ldots \ldots \ldots \ldots \ldots \ldots \ldots \ldots \ldots \ldots \ldots \ldots \ldots$ & 0.0137 & yes & 3 & 4 & $\cdots$ & 490 & 27 & 45.87 & -3.0 & 1 \\
\hline 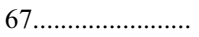 & 0.0245 & yes & 2 & 4 & 14 & 363 & 49 & 46.03 & 0.0 & -8 \\
\hline 68.................... & 0.0080 & yes & 3 & 5 & $\ldots$ & 263 & 33 & 45.47 & -1.0 & -9 \\
\hline $74 \ldots \ldots \ldots \ldots \ldots \ldots \ldots$ & 0.0399 & $\ldots$ & 4 & 5 & $\ldots$ & 537 & 39 & 46.32 & -4.2 & 2 \\
\hline $79 \ldots \ldots \ldots \ldots \ldots \ldots \ldots$ & 0.0145 & $\ldots$ & 4 & $\ldots$ & $\ldots$ & 229 & 7 & 44.83 & -0.2 & -10 \\
\hline 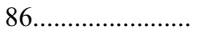 & 0.0199 & yes & 4 & $\ldots$ & $\ldots$ & 457 & 47 & 46.20 & -3.5 & 0 \\
\hline 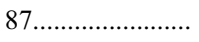 & 0.0296 & $\cdots$ & 3 & $\cdots$ & 6 & 145 & 31 & 44.91 & 3.0 & 1 \\
\hline $88 \ldots \ldots \ldots \ldots \ldots \ldots \ldots$ & 0.0201 & $\ldots$ & 2 & 4 & 6 & 27 & 68 & $\ldots$ & 4.0 & -18 \\
\hline $89 \ldots \ldots \ldots \ldots \ldots \ldots \ldots$ & 0.0297 & $\ldots$ & 2 & 4 & $\ldots$ & 52 & 59 & 44.86 & 5.0 & -20 \\
\hline $90 \ldots \ldots \ldots \ldots \ldots \ldots \ldots \ldots \ldots \ldots \ldots \ldots \ldots$ & 0.0088 & yes & 4 & $\ldots$ & 9 & 166 & 30 & 45.00 & 1.8 & -5 \\
\hline $93 \ldots \ldots \ldots \ldots \ldots \ldots$ & 0.0168 & $\ldots$ & 4 & $\ldots$ & $\ldots$ & 355 & 71 & 46.10 & 0.0 & -5 \\
\hline 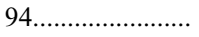 & 0.0417 & $\ldots$ & 5 & 7 & $\ldots$ & 832 & 58 & 46.72 & -3.5 & 5 \\
\hline $98 \ldots \ldots \ldots \ldots \ldots \ldots \ldots$ & 0.0266 & $\ldots$ & 2 & 3 & $\ldots$ & 204 & 28 & 45.35 & -2.0 & 6 \\
\hline
\end{tabular}

a From Hickson (1982).

b From Ponman et al. (1996).

c From Ribeiro et al. (1998). 
TABLE 6

EfFect Expected of InCOMPleteness on Our AnAlysis

\begin{tabular}{|c|c|c|c|c|c|c|}
\hline $\mathrm{HCG}$ & Missing Galaxies & Morphology & $\left\langle\mathrm{EW}_{\mathrm{act}}\right\rangle$ & Changes & $\langle T\rangle$ & $\langle T\rangle_{C}$ \\
\hline .............. & $\mathrm{c}$ & E2 & -199 & + & 1.7 & 0.0 \\
\hline 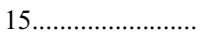 & $e, f$ & $\mathrm{Sa}, \mathrm{Sbc}$ & 0 & 0 & -2.4 & -0.1 \\
\hline $23 \ldots \ldots \ldots \ldots \ldots \ldots \ldots$ & $\mathrm{b}$ & $\mathrm{Sc}$ & -73 & - & 2.3 & 3.0 \\
\hline $30 \ldots \ldots \ldots \ldots \ldots \ldots \ldots$ & $\mathrm{c}, \mathrm{d}$ & $\mathrm{SBc}, \mathrm{S} 0$ & 3 & 0 & 3.0 & 2.0 \\
\hline 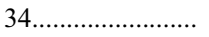 & $\mathrm{b}, \mathrm{d}$ & $\mathrm{Sd}, \mathrm{S} 0$ & -49 & 0 & 0.5 & 1.5 \\
\hline 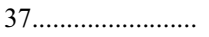 & $\mathrm{e}$ & E0 & -11 & + & 2.2 & 1.4 \\
\hline 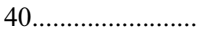 & $\mathrm{c}$ & Sbc & -10 & - & -0.2 & 0.6 \\
\hline $42 \ldots \ldots \ldots \ldots \ldots \ldots \ldots \ldots$ & $\mathrm{d}$ & $\mathrm{E}$ & 2 & + & -4.0 & -4.2 \\
\hline $62 \ldots \ldots \ldots \ldots \ldots \ldots \ldots$ & $\mathrm{d}$ & $\mathrm{E}$ & 1 & + & -3.0 & -3.5 \\
\hline 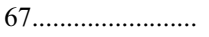 & $\mathrm{c}, \mathrm{d}$ & Scd, S0 & -8 & 0 & 0.0 & 1.0 \\
\hline $68 \ldots \ldots \ldots \ldots \ldots \ldots \ldots$ & $\mathrm{d}, \mathrm{e}$ & E, S0 & -9 & + & -1.0 & -2.0 \\
\hline $74 \ldots \ldots \ldots \ldots \ldots \ldots \ldots \ldots$ & $\mathrm{e}$ & S0 & 2 & + & -4.2 & -3.8 \\
\hline $88 \ldots \ldots \ldots \ldots \ldots \ldots \ldots \ldots$ & $\mathrm{a}, \mathrm{d}$ & $\mathrm{Sbc}, \mathrm{Sc}$ & -18 & - & 4.0 & 4.2 \\
\hline 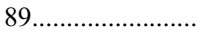 & $\mathrm{c}, \mathrm{d}$ & Scd, Sm & -20 & - & 5.0 & 6.2 \\
\hline 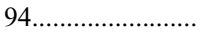 & $\mathrm{e}, \mathrm{f}, \mathrm{g}$ & $\mathrm{Sd}, \mathrm{S} 0, \mathrm{~S} 0$ & 5 & + & -3.5 & -1.6 \\
\hline $98 \ldots \ldots \ldots \ldots \ldots \ldots \ldots \ldots \ldots \ldots \ldots \ldots$ & $\mathrm{c}$ & $\mathrm{E}$ & 6 & + & -2.0 & -0.8 \\
\hline
\end{tabular}

Finally, according to Ribeiro et al. (1998), HCG 4 was misclassified as a CG. They showed that galaxies HCG 4e and HCG $4 \mathrm{c}$ were not group members. Eliminating them causes HCG 4 to fail the definition for a compact group according to Hickson.

Table 7 summarizes our classification for all the groups in our sample (with the exception of HCG 4). In this table, we also list the mean characteristics associated with the different types. In columns (2) and (3), a colon following the number identifies groups with deviant or marginally deviant dynamical characteristics (for example, HCG 34 and 37 have significantly higher velocity dispersions than the other groups belonging to type B).

Not considering the exceptions, the CGs in the different main configuration are clearly separated in Figure 9. HCGs

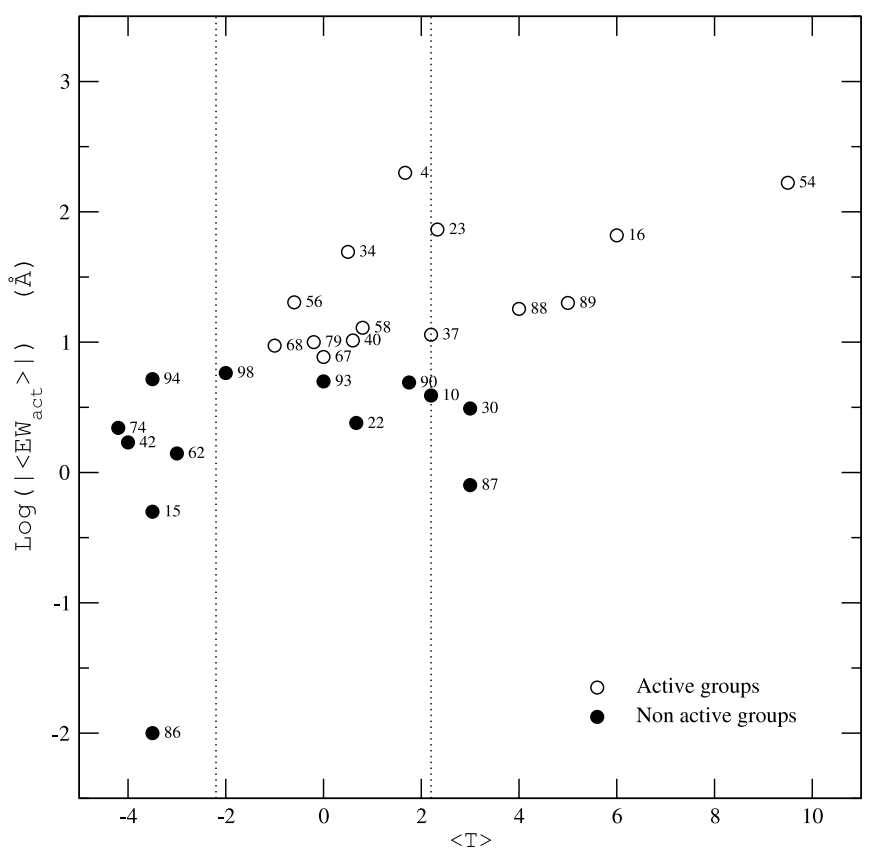

FIG. 9.-Mean activity index as a function of mean morphology. The CGs are identified by their HCG number. The vertical dotted lines separate the groups according to the three main spatial (projected) configurations. The evolution of groups increases as $\mathrm{A} \Rightarrow \mathrm{B} \Rightarrow \mathrm{C}$. with a mean $T>2.2$ are all type A systems. Those with a mean $T<-2.2$ are all of type C. Transition groups are classified as type B. In terms of evolution, we may distinguish a continuous sequence, which goes $\mathrm{A} \Rightarrow \mathrm{B} \Rightarrow \mathrm{C}$, with $\mathrm{A}$ less evolved than $\mathrm{C}$. We emphasize that in terms of spatial configuration alone, such an evolutionary sequence is not obvious (considering also the exceptions), and one needs a second parameter, such as the activity index introduced in this paper, for it to become noticeable. This may explain why the different configuration types were not recognized before.

Using a one-way ANOVA test, we confirm the differences observed between the characteristics of types A, B, and C, as shown in Table 7, at a 95\% confidence level. In Table 8, we show the results of Tukey's multiple test, which allows us to identify the source of the variance. ${ }^{3}$ This table reports the $P$ values for the difference between each pair of mean values. If the null hypothesis is true (all the values are sampled from populations with the same mean), then there is only a $5 \%$ chance that any one or more comparisons will have a $P$ value less than 0.05 . These tests confirm the different evolutionary level of the three types defined in this work.

\subsection{The Relation between Galaxy Activity and the Dynamics of CGs}

According to the conventional scenario for the fate of CGs, also referred to as the fast merger model (Mendes de Oliveira \& Hickson 1994; Gómez-Flechoso \& Domínguez-Tenreiro 2001), young forming groups are expected to start out with high velocity dispersions and large radii, which would rapidly decrease as the galaxies merge to form one giant elliptical (this is illustrated by the model CG2 in Figure 1 of Gómez-Flechoso \& Domínguez-Tenreiro 2001). Assuming the groups form from spiral galaxies that originated in the field, we would thus expect young groups to be rich in late-type galaxies and to become gradually richer in early-type galaxies before their final merging phase. In terms of activity, we would also expect the level of star formation and AGN to be high at the beginning of the sequence and to decrease gradually, maybe with a change from star formation to AGN, in the final merger phase.

\footnotetext{
${ }^{3}$ One-way ANOVA with Tukey's post test was performed using GraphPad Prism version 3.00 for Windows, from GraphPad Software, San Diego, CA (http://www.graphpad.com).
} 

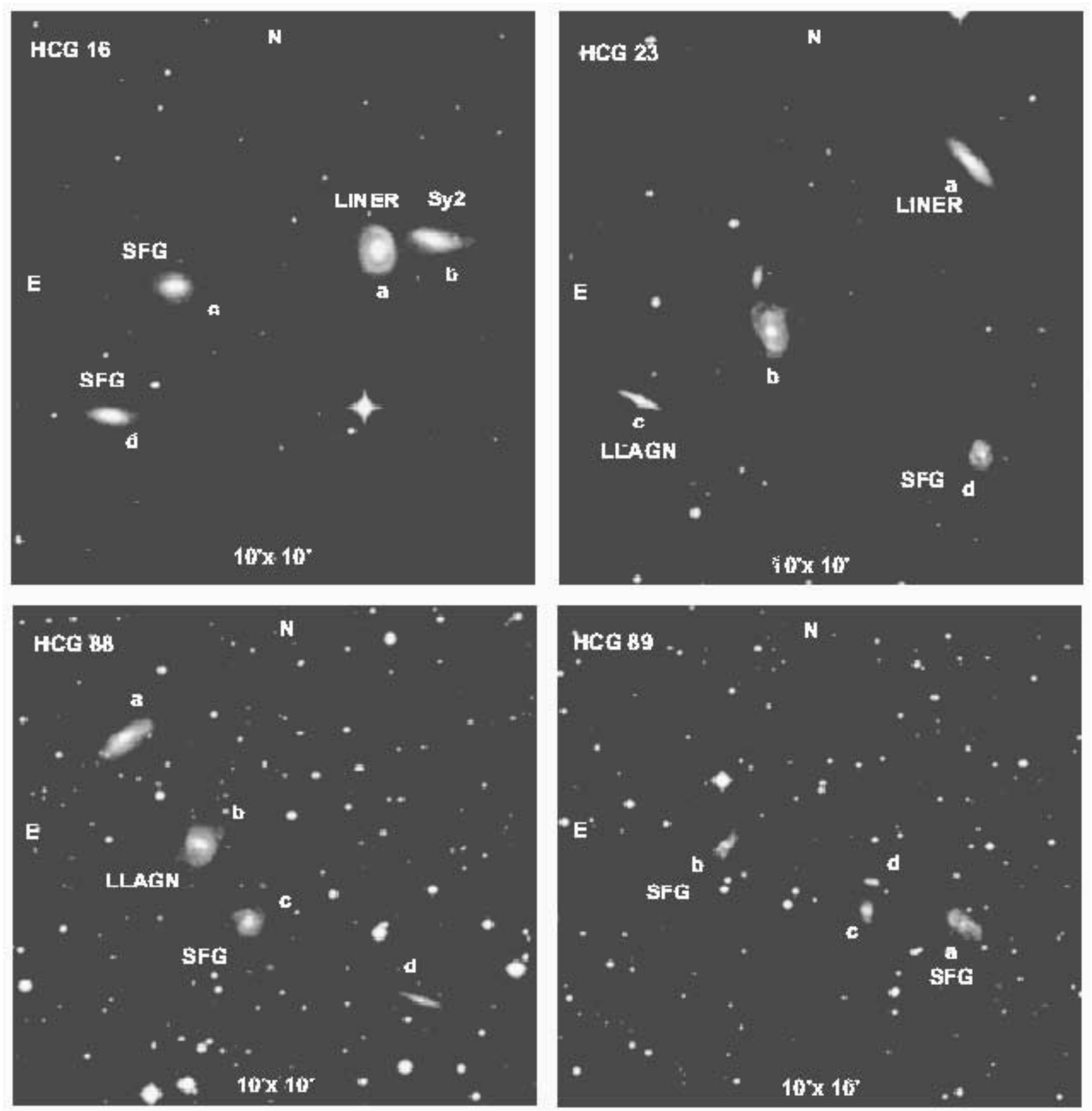

Fig. 10.-Type A prototypes: HCG 16, 23, 88, and 89. The letters identifying individual group members follow the notation given by Hickson (1982). The activity types from Table 2 are indicated next to their respective galaxy. The angular size of each frame is indicated along the bottom. 

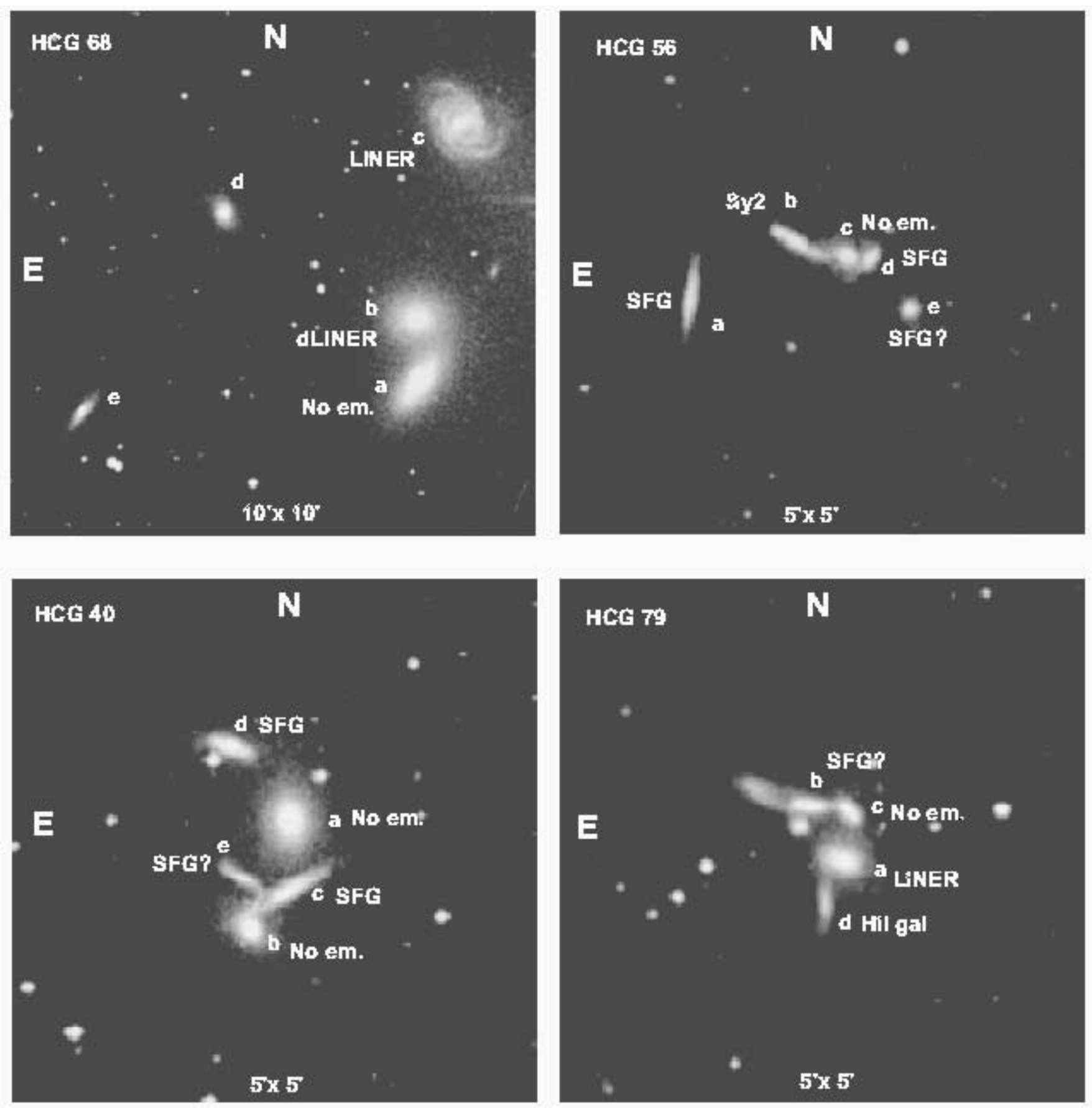

FIG. 11.-Type B prototypes: HCG 40, 56, 68, and 79. Same specifications as in Fig. 10. 


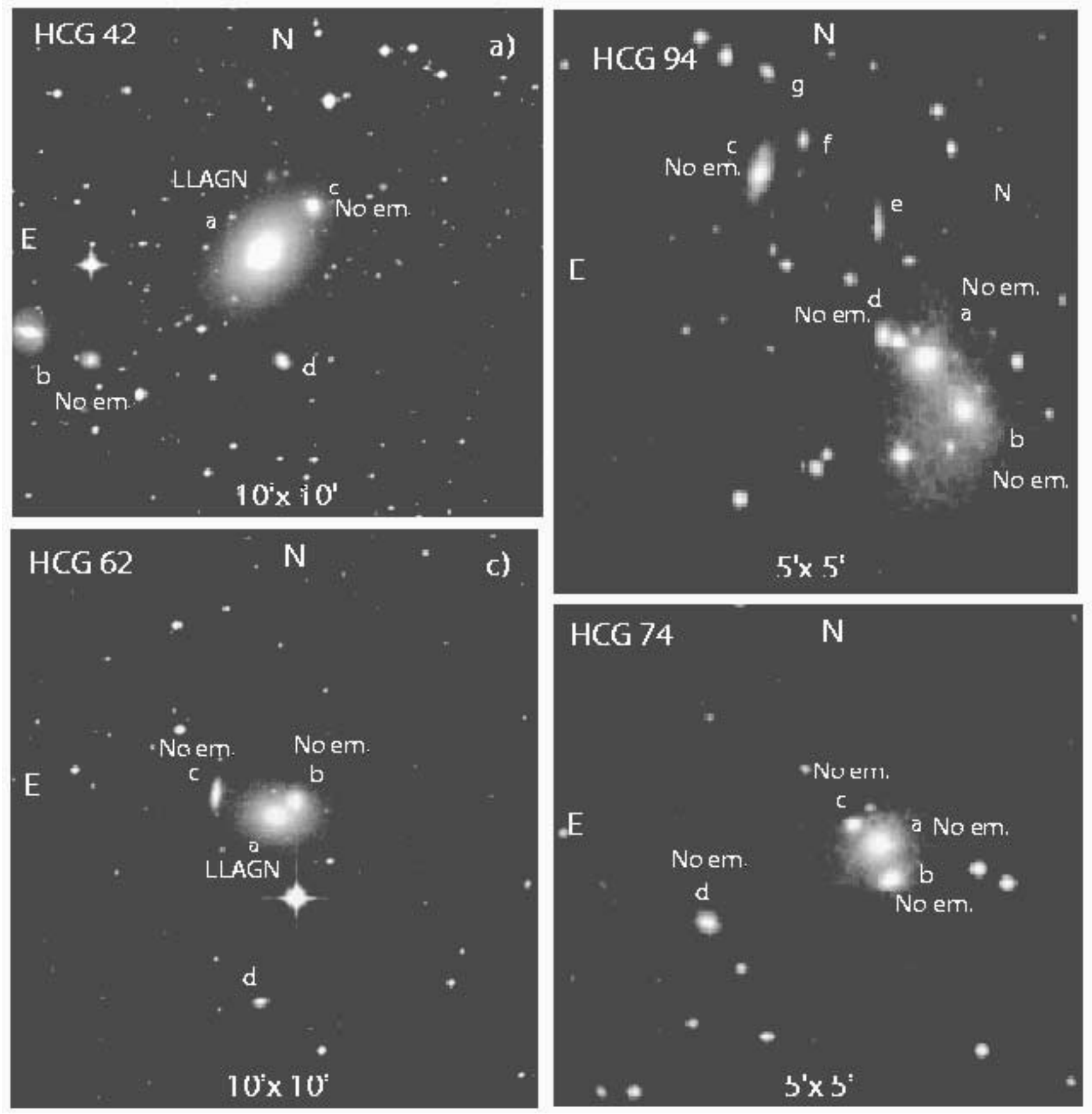

FIG. 12.-Type C prototypes, in which one giant elliptical dominates the group: HCG 42, 62, 74, and 94. Same specifications as in Fig. 10. 

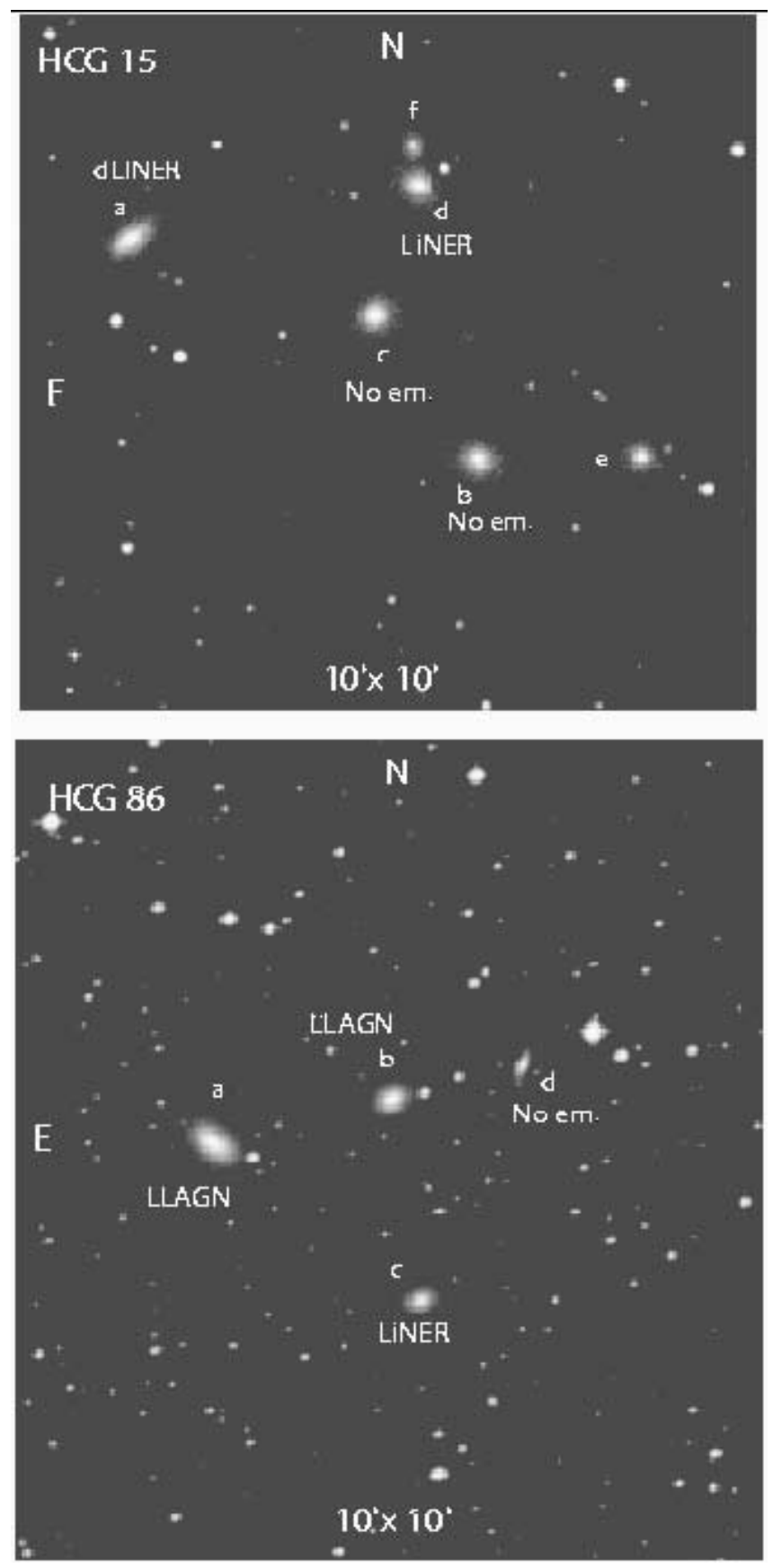

Fig. 13.-Type C prototypes, which are dominated by several elliptical galaxies: HCG 15 and 86. Same specifications as in Fig. 10.
According to the conventional fast merger model for CGs, we would thus expect to see an evolutionary sequence that would trace a very specific pattern in a graph mapping the radius as a function of the velocity dispersion. The expected pattern is sketched in Figure 14a. The actual observed positions are shown in Figure $14 b$. One can immediately see that the expected pattern is not reflected by the observations: CGs in type A, which are less evolved, should have large radii and high velocity dispersions, whereas CGs in type $\mathrm{C}$, which are more evolved, should have small radii and low velocity dispersions. Contrary to expectation, type $\mathrm{C}$ CGs have radii comparable to those of type A and much higher velocity dispersions (see Table 7).

In Figure 15 we compare the mean activity index and morphology with the radius of the groups (HCG 54 was omitted). We find no relation between activity or morphology and the radius of the group. The mean activity index and morphology are compared with the velocity dispersion in Figure 16. We distinguish no relation between the activity and the velocity dispersion. However, we see a clear trend for groups dominated by early-type galaxies to have high velocity dispersions. In terms of evolution, we see the velocity dispersion increasing from type A to $\mathrm{C}$ (see also Tables 7 and 8 ), which indicates that the most evolved groups have the highest velocity dispersions.

The trend we observe in our sample between evolution and velocity dispersion seems to stem from the strong correlation between morphology and velocity dispersion previously discovered by Hickson et al. (1988). Our observations support their claim that the general relation between morphology and velocity dispersion encountered in CGs is an evolutionary phenomenon linked to the environment (for other evidence in favor of this interpretation, see Shaker et al. 1998 and Focardi \& Kelm 2002).

\section{DISCUSSION}

The trends we observe in our sample are, at best, only partially consistent with the conventional fast merger model for CGs. We do distinguish what could be young forming groups (type A) and more evolved merging groups (type B). However, the velocity dispersions and radii of these systems are contrary to what is expected. In a sense, this negative result is somewhat discomforting, because it suggests that the dynamical evolution of galaxies in CGs is more complex than previously believed. However, this result does not have to be too surprising, since this is what one expects if CGs are in reality part of larger scale structures (Rose 1977; West 1989; White 1990; Diaferio et al. 1994; Governato et al. 1996).

Assuming the formation and evolution processes of CGs are related to larger scale structures, we may have found a new

TABLE 7

Configuration Types and Mean Properties

\begin{tabular}{|c|c|c|c|c|c|c|c|}
\hline \multirow[b]{2}{*}{$\begin{array}{l}\text { TyPE } \\
(1)\end{array}$} & \multicolumn{2}{|c|}{ HCG Group Number } & \multirow{2}{*}{$\begin{array}{c}\langle\text { Velocity DisPERSION }\rangle \\
\left(\mathrm{km} \mathrm{s}^{-1}\right) \\
(4)\end{array}$} & \multirow{2}{*}{$\begin{array}{c}\langle\text { RADIUS }\rangle \\
(\mathrm{kpc}) \\
(5)\end{array}$} & \multirow{2}{*}{$\begin{array}{c}\langle M\rangle \\
(\mathrm{g}) \\
(6)\end{array}$} & \multirow[b]{2}{*}{$\begin{array}{l}\langle T\rangle \\
(7)\end{array}$} & \multirow{2}{*}{$\begin{array}{c}\langle E W\rangle \\
(\AA) \\
(8)\end{array}$} \\
\hline & $\begin{array}{c}\text { Active } \\
\text { (2) }\end{array}$ & $\begin{array}{c}\text { Inactive } \\
\text { (3) }\end{array}$ & & & & & \\
\hline $\mathrm{A} \ldots \ldots \ldots \ldots \ldots \ldots \ldots$ & $16,23,88,89$ & 30,87 & $135 \pm 38$ & $53 \pm 6$ & $45.2 \pm .2$ & $3.9 \pm 0.6$ & $-29 \pm 13$ \\
\hline $\mathrm{B} \ldots \ldots \ldots \ldots \ldots \ldots$ & $37:, 34:, 40,56,67,68,79$ & $22,90,98$ & $302 \pm 61$ & $25 \pm 4$ & $45.3 \pm .2$ & $0.2 \pm 0.4$ & $-11 \pm 5$ \\
\hline $\mathrm{C} \ldots \ldots \ldots \ldots \ldots \ldots \ldots$ & $\ldots$ & $15,42,62:, 86,74,94$ & $567 \pm 72$ & $49 \pm 7$ & $46.2 \pm .2$ & $-3.6 \pm 0.2$ & $2 \pm 1$ \\
\hline Exceptions....... & $10,54,4$ & 58,93 & $\ldots$ & $\ldots$ & $\ldots$ & $\ldots$ & $\ldots$ \\
\hline
\end{tabular}


TABLE 8

Results for Tukey's Post Tests

\begin{tabular}{|c|c|c|c|c|c|}
\hline Types & $\langle$ Velocity Dispersion $\rangle$ & $\langle$ Radius $\rangle$ & $\langle M\rangle$ & $\langle T\rangle$ & $\langle E W\rangle$ \\
\hline $\mathrm{A}-\mathrm{B} \ldots \ldots \ldots \ldots \ldots$ & $>0.05$ & $<0.01$ & $>0.05$ & $<0.001$ & $>0.05$ \\
\hline $\mathrm{A}-\mathrm{C} \ldots \ldots \ldots \ldots \ldots$ & $<0.001$ & $>0.05$ & $<0.05$ & $<0.001$ & $<0.05$ \\
\hline $\mathrm{B}-\mathrm{C} \ldots \ldots \ldots \ldots \ldots$ & $<0.05$ & $<0.05$ & $<0.05$ & $<0.001$ & $>0.05$ \\
\hline
\end{tabular}

clue how to explain their behavior by relating the evolutionary level of a group to its velocity dispersion. Indeed, in dynamical terms, the velocity dispersion generally increases with the total mass of the system (Heisler et al. 1985; Perea et al. 1990). By applying this observation to CGs, we may thus conclude that their level of evolution increases with their mass, or more properly, with the mass of the structure with which they are associated or where they are embedded in.

The above interpretation is supported by the three following independent observations. First, we note in Table 7 that the mean masses of the groups, as defined by Hickson (see Tables 4 and 5), does increase from types A to C. This is confirmed by a one-way ANOVA test at a 95\% confidence level. In Table 8, the results of Tukey's multiple test confirm that groups in type $\mathrm{C}$ are more massive than those in type $\mathrm{A}$ and $\mathrm{B}$. Second, we note that the number of galaxies in structures associated with
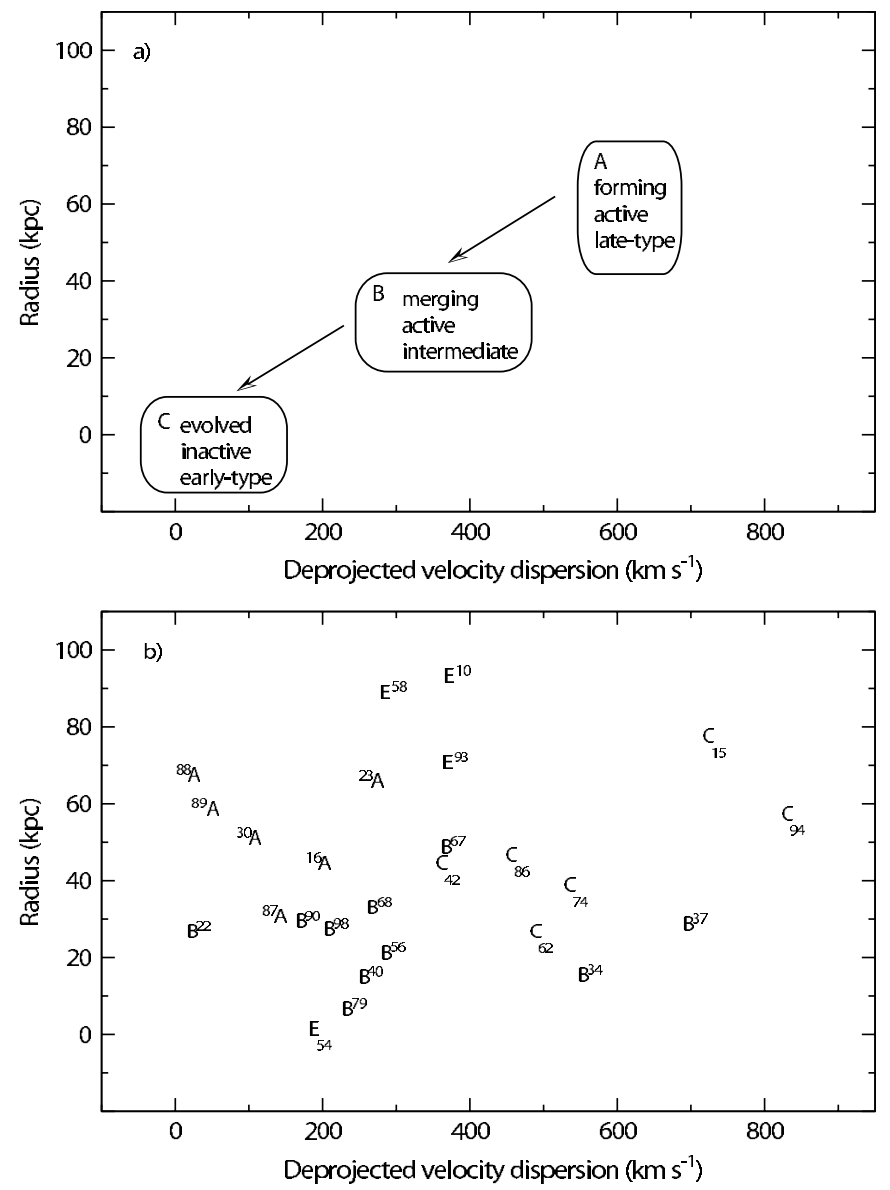

FIG. 14.- (a) Illustration of the expected evolutionary pattern, according to the conventional fast merger model for CGs, in a diagram of radius vs. velocity dispersion; $(b)$ actual positions occupied by the 26 CGs (excluding HCG 4) in our sample. The CGs are identified by their HCG number; the letters correspond to the configuration types. The E means an exception (no classification is possible).
CGs generally increases with the velocity dispersion. Looking at the number of galaxies encountered by Ribeiro et al. (1998) in Table 4 and 5 , we see that the structures associated with groups of type A contain between six and eight galaxies, whereas those associated with groups in type $\mathrm{C}$ contain between nine and 18 galaxies (the only exception being HCG 22). The third observation is the detection of diffuse X-ray envelopes. Naturally, one expects these envelopes to be more common or easily detected in massive systems. Using the data in Tables 4 and 5, we see that only one nonevolved group with low velocity dispersion (lower than $300 \mathrm{~km} \mathrm{~s}^{-1}$ ) has an X-ray envelope. This is HCG 16 (type A), which is unusually active (see Ribeiro et al. 1996). Another small-velocity-dispersion group with an X-ray envelope is the relatively evolved group HCG 58. But, this group may be considered unusually active as well, since it contains the only Seyfert 1 galaxy in our sample. All the other X-ray envelopes are detected primarily in type C HCG 15 , $42,62,86$, and 90 , and type B HCG 37, 67, and 68. With the exception of HCG 90, all these groups have velocity dispersions higher than $300 \mathrm{~km} \mathrm{~s}^{-1}$.

Assuming that the velocity dispersion increases with the mass of the structure, a ready interpretation of our observations is that CGs associated with massive structures are more evolved. Theoretically, it is easy to find arguments in favor of this interpretation. For example, if we assume that a large number of interactions accelerates the evolution of galaxies, then we would naturally expect galaxies in more massive and rich systems to be more evolved. However, tidal interactions become less effective as collision velocities increase, which then seems to contradict what we observe. Alternatively, if the formation of CGs follows the formation of large-scale structures then, under certain conditions, we would expect highdensity structures to collapse before low-density ones, forming primordially massive structures, and CGs associated with such structures to be naturally older.

Our observations are consistent with the formation of structures as predicted by the CDM model (or $\Lambda \mathrm{CDM}$ ), assuming the formation of galaxies is a biased process (Davis et al. 1985; Benson et al. 2001), that is, galaxies develop first in high-density structures. In terms of evolution, biased galaxy formation would naturally lead to more massive systems being more evolved (see Fig. 1 in Benson et al. 2001). If one follows the CDM model evolution over time, galaxies are seen to form first in the high-density structures, then structures are seen to get richer and more complex through mergers of smaller systems. As part of the structure formation process, the evolution of small-scale entities like CGs must consequently be more complex than predicted by the conventional fast merger model. This is because the galaxies in these groups are possibly influenced not only by the other galaxy members but also by the neighboring structures (see Einasto et al. 2003; Ragone et al. 2004).

As part of the structure formation process, the fate of CGs may also be different than that predicted by the conventional fast merger model. Since structures continuously merge to 

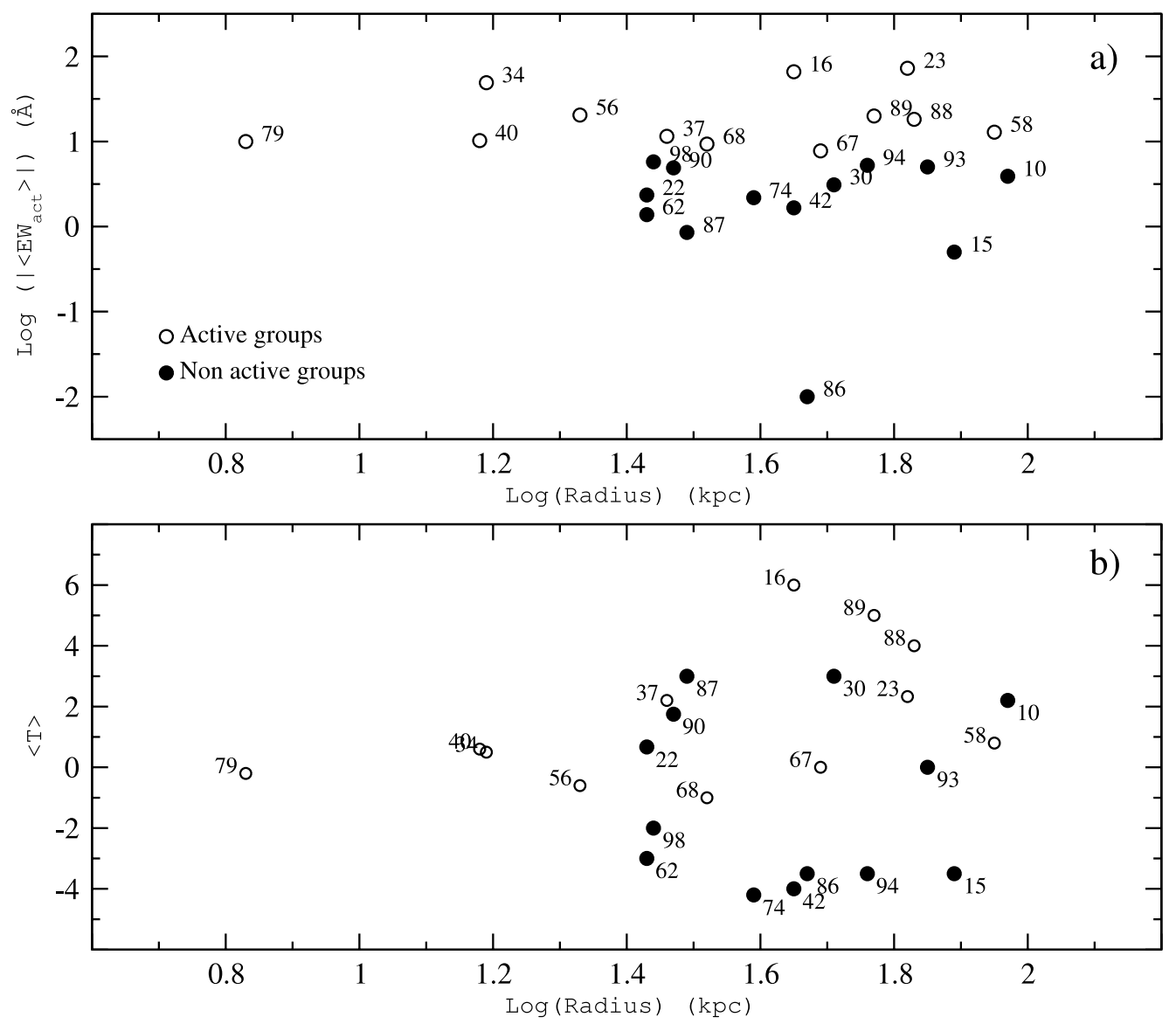

FIG. 15.-(a) Mean activity index as a function of radius. (b) Mean morphological type as a function of radius. 

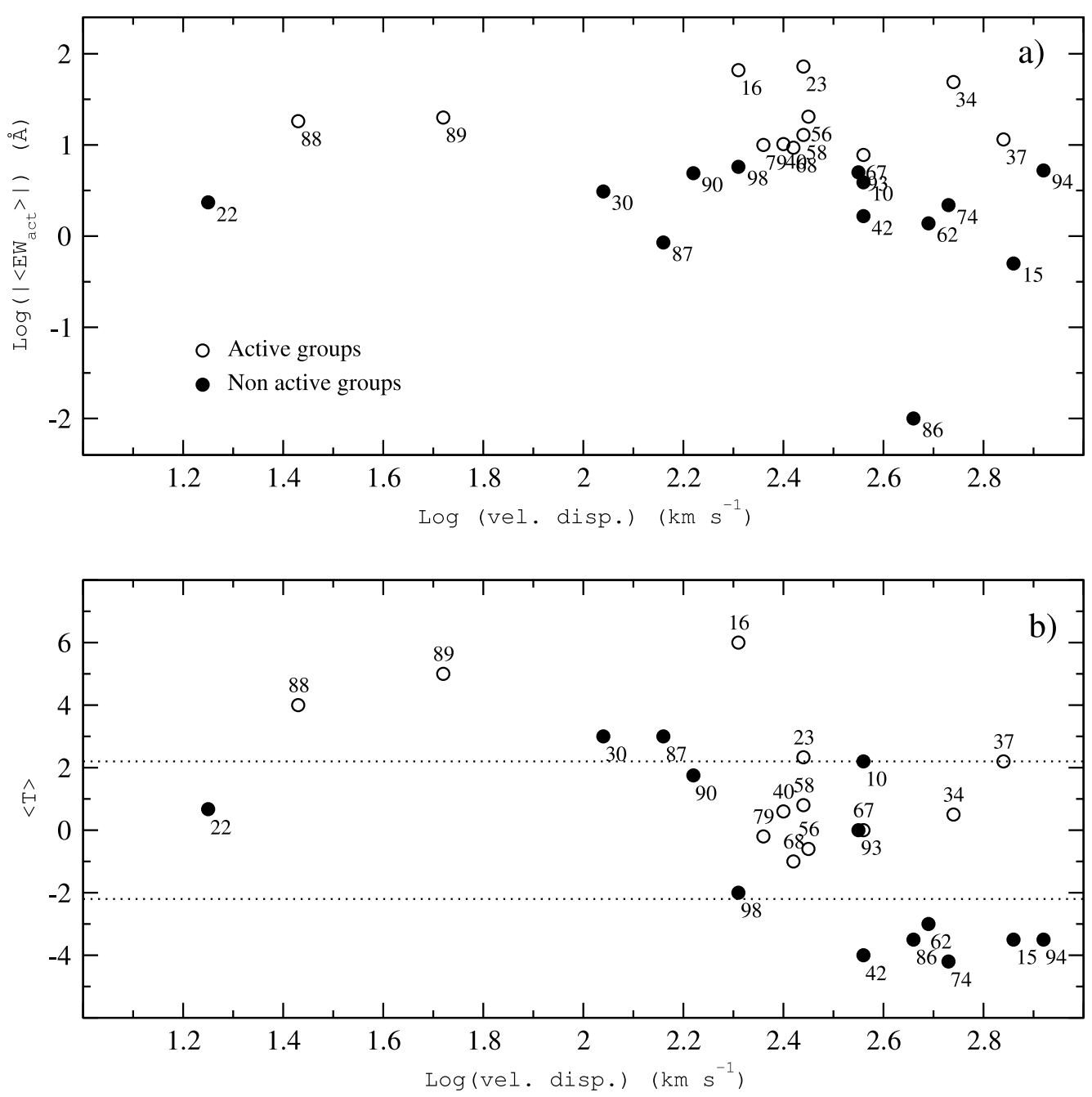

FIG. 16. - (a) Mean activity index as a function of radius. (b) Mean morphological type as a function of radius. The two dotted lines separates the group with different configuration types and evolutionary states (see Fig. 9).

form larger ones, the first groups to form (type C), being in denser environments, would already have had time to merge with other similarly evolved groups. The most evolved groups would thus be expected today to form part of larger structures where they would be in dynamical equilibrium.

As for the groups that seem to have formed more recently (type A) or those that formed in the recent past (type B), the galaxies in these groups obviously have not had enough time to merge. Being found in less massive environments, it is not clear whether they will experience a complete merger of their components or whether they will find other groups with which they can merge to form larger structures (and reach equilibrium before completing the merger of their members). Such a scenario may explain some of the exceptions to the trends found in our sample, like HCG 54 (and maybe HCG 31), as an example of a group in a low-density environment that is completing a merger, or HCG 10, 58 and 93, as examples of different groups merging together to form a larger structure.

Finally, it is important to note that we can possibly distinguish between the two hypotheses suggested to explain our observations by examining CGs at higher redshifts. In particular, if the structure formation hypothesis is correct, one would thus expect to find CGs at higher redshift associated with more massive structures than those at lower redshift.

\section{SUMMARY AND CONCLUSIONS}

We have defined a new spectroscopic index that allows us to quantify the level of activity (AGN and star formation) in galaxies. By taking the mean of this index in a CG and combining it with the mean morphological type of the galaxies, quantifying the evolutionary state of these systems is possible.

Applying our method to a sample of 27 CGs from Hickson's catalog, we have found an evolutionary sequence, that correlates with the projected spatial configuration of the groups. Mapping the position of CGs with different evolutionary states and group morphologies in a diagram of radius versus velocity dispersion, we did not observe the pattern predicted by the conventional fast merger model. Contrary to expectation, we found that the level of evolution of CGs increases with velocity dispersion. This trend was further shown to be possibly connected to the masses of the CGs or to the structures in which they are embedded. Assuming that the velocity dispersion increases with the mass, the trend we observe would thus imply that the most evolved groups are found within the most massive structures.

We propose two different hypotheses to explain our results. The first assumes that the evolution of galaxies accelerates 
with the number of interactions in massive structures. The other assumes that the formation of CGs follows the formation of large-scale structure, and that massive structures develop before less massive ones. Our observations are consistent with structure formation as predicted by the CDM model (or $\Lambda \mathrm{CDM}$ ), assuming that galaxy formation is a biased process with galaxies developing first in high-density structures.

The authors would like to thank the Time Allocation Committee of the Observatorio Astronómico Nacional at San
Pedro Mártir for generous allocations of observing time, which has made this project possible. We also would like to thank the support personnel of the observatory, and in particular the two night assistants Felipe Montalvo Rocha and Salvador Monroy, without whom the observations would have been arduous, to say the least. R. C. is grateful to Heinz Andernach for reading and commenting on an early version of the manuscript. The referee, Paul Hickson, is also acknowledged for his comments and suggestions that helped improve our analysis and discussion. This research was supported in part by CONACyT grants EX-000479 and 40194.
Aceves, H., \& Velázquez, H. 2002, Rev. Mexicana Astron. Astrofis., 38, 199

Barbaro, G., \& Poggianti, 1997, A\&A, 324, 490

Barnes, J. E. 1989, Nature, 338, 123

Barton, E. J., de Carvalho, R. R., \& Geller, M. J. 1998, AJ, 116, 1573

Benson, A. J., Frenk, C. S., Baugh, C. M., Cole, S., \& Lacey, C. G. 2001, MNRAS, 327, 1041

Byrd, G., \& Valtonen, M. 1990, ApJ, 350, 89

Contini, T., Considère, S., \& Davoust, E. 1998, A\&AS, 130, 285

Copetti, M. V. F., Pastoriza, M. G., \& Dottori, H. A. 1986, A\&A, 156, 111

Coziol, R. 1996, A\&A, 309, 345

Coziol, R., de Carvalho, R. R., Capelato, H. V., \& Ribeiro, A. L. B. 1998a, ApJ, 506, 545

Coziol, R., Iovino, A., \& de Carvalho, R. R. 2000, AJ, 120, 47

Coziol, R., Ribeiro, A. L. B., de Carvalho, R. R., \& Capelato, H. V. 1998b, ApJ, 493, 563

Davis, M., Efstathiou, G., Frenk, C. S., \& White, S. D. M. 1985, ApJ, 292, 371

Diaferio, A., Geller, M. J., \& Ramella, M. 1994, AJ, 107, 868

Einasto, M., Einasto, J., Müller, V., Heimämäki, P., \& Tucker, D. L. 2003, A\&A, 401, 851

Focardi, P., \& Kelm, B. 2002, A\&A, 391, 35

Fujita, Y. 1998, ApJ, 509, 587

Garcia, A. M. 1995, A\&A, 297, 56

Gómez-Flechoso, M. A., \& Domínguez-Tenreiro, R. 2001, ApJ, 549, L187

Governato, F., Tozzi, P., \& Cavaliere, A. 1996, ApJ, 458, 18

Heisler, J., Tremaine, S., \& Bahcall, J. N. 1985, ApJ, 298, 8

Henriksen, M. J., \& Byrd, G. 1996, ApJ, 459, 82

Hickson, P. 1982, ApJ, 255, 382

Hickson, P., Kindl, E., \& Huchra, J. P. 1988, ApJ, 331, 64

Ho, L. 1999, Adv. Space Res., 23, 813

\section{EFERENCES}

Kennicutt, R. C., Jr. 1992, ApJS, 79, 255

Kennicutt, R. C., Jr., \& Kent, S. M. 1983, AJ, 88, 1094

Lawrence, A. 1999, Adv. Space Res., 23, 1167

Leonardi, A. J., \& Rose, J. A. 1996, AJ, 111, 182

Mendes de Oliveira, C., \& Hickson, P. 1994, ApJ, 427, 684

Merritt, D. 1983, ApJ, 264, 24 . 1984, ApJ, 276, 26

Miller, N. A., \& Owen, F. N. 2002, AJ, 124, 2453

Osterbrock, D. E. 1981, ApJ, 249, 462

Perea, J., del Olmo, A., \& Moles, M. 1990, A\&A, 237, 319

Ponman, T. J., Bourner, P. D. J., Ebeling, H., \& Böhringer, H. 1996, MNRAS, 283, 690

Ragone, C. J., Merchán, M., Muriel, H., \& Zandivarez, A. 2004, MNRAS, in press (astro-ph/0402155)

Ramella, M., Diaferio, A., Geller, M. J., \& Huchra, J. P. 1994, AJ, 107, 1623

Ribeiro, A. L. B., de Carvalho, R. R., Capelato, H. V., \& Zepf, S. E. 1998, ApJ, 497, 72

Ribeiro, A. L. B., de Carvalho, R. R., Coziol, R., Capelato, H. V., \& Zepf, S. E. 1996, ApJ, 463, L5

Rood, H. J., \& Struble, M. F. 1994, PASP, 106, 413

Rose, J. A. 1977, ApJ, 211, 311

- 1985, AJ, 90, 1927

Severgnini, P., Garilli, B., Saracco, P., \& Chincarini, G. 1999, A\&AS, 137, 495

Shaker, A. A., Longo, G., \& Merluzzi, P. 1998, Astron. Nachr., 319, 167

Turner, M. J. L., et al. 2001, A\&A, 365, L110

Veilleux, S., \& Osterbrock, D. E. 1987, ApJS, 63, 295

West, M. J. 1989, ApJ, 344, 535

White, S. D. M. 1990, in Dynamics and Interactions of Galaxies, ed. R. Weilen (New York: Springer), 380 